\title{
Risk and Valuation of Mortality Contingent Catastrophe Bonds*
}

\author{
Daniel Bauer ${ }^{\dagger}$ \\ DFG Research Training Group 1100, Ulm University \\ Helmholtzstraße 18, 89069 Ulm, Germany \\ Florian W. Kramer \\ DFG Research Training Group 1100, Ulm University \\ Helmholtzstraße 18, 89069 Ulm, Germany
}

July 2007

\begin{abstract}
Catastrophe Mortality Bonds are a recent capital market innovation providing insurers and reinsurers with the possibility to transfer catastrophe mortality risk off their balance sheets to capital markets.

This article introduces a time-continuous model for analyzing and pricing catastrophe mortality contingent claims based on stochastic modeling of the force of mortality. In addition, we give an concise survey of past transactions and explain in detail the structure of the deals and the securities.

Parametrizations of the proposed model based on three different calibration procedures are derived. The resulting loss profiles and prices are compared to loss profiles provided by the issuers and to market prices, respectively. We find that the profiles are subject to great uncertainties and should hence be considered with care by investors and rating agencies. Furthermore, by comparing outcomes of risk-adjusted parametrizations based on insurance quotes and parametrizations implied by market prices, we are able to give a possible explanation for the relatively fast growth of the market for Catastrophe Mortality Bonds over the last years.
\end{abstract}

JEL classification: G12; G20; C60

Keywords: Insurance Securitization; Catastrophe Bonds; Stochastic Mortality Modeling

\footnotetext{
${ }^{*}$ The authors thank Heiko Logisch (Commerzbank AG), Rüdiger Kiesel (Ulm University), and Norbert Renz (Lebensversicherung von 1871 a.G.) for their support and helpful discussions. Furthermore, we are grateful to Gunter Löffler, Cora Nohrden, Andreas Reuss, and Christian Schmidt for valuable comments. The usual disclaimer applies.

${ }^{\dagger}$ Corresponding author. Phone: +49 (731) 50 31088. Fax: +49 (731) 50 23548. E-mail addresses: daniel.bauer@uni-ulm.de (D. Bauer); florian.kramer@uni-ulm.de (F. W. Kramer)
} 


\section{Introduction}

Regulatory changes such as the Solvency II requirements of the European Union or Phase II of the International Financial Reporting Standard 4 demand new valuation methodologies for insurance liabilities. In particular, emphasis is put on stochastic models for the evolution of the insurer's investments. Moreover, complex financial options offered within insurance products, such as guaranteed minimum benefits within variable annuities or interest rate guarantees within conventional products, together with an increasing competition from banks, mutual funds, and other financial service providers ask for more advanced valuation and risk management techniques than the "classical actuarial toolbox" offers. Consequently, capital markets and insurance markets converge as actuaries and capital market investors are accepting a common language and, more importantly, common definitions and management strategies for risk (see e.g. Culp (2006)).

Aside from applying similar valuation methodologies, insurers have picked up some other ideas from the banking world regarding the management of their liabilities: "I don't see mortgage risk in banks' balance sheets. I see them give the service, take the fees and offload the risk. We need to develop that model in the future because it will make the industry less capital intensive, both life and non-life." (Henri de Castries, Chairman of the management board and CEO of the AXA group). By means of securitization, i.e. isolating the cash flows that are linked to insurance liabilities and repackaging them into cash flows that are traded in capital markets, insurers and reinsurers can step away from their traditional risk warehousing function towards a business model of risk intermediation. This way, the insurance industry can operate more efficiently as well as cover the increasing demand for insurance from developing countries (cf. Cowley and Cummins (2005)). Consequently, in recent years, efforts to securitize insurance risks have increased. However, the market for Insurance Linked Securities (ILS) is still small in comparison to the total volume of risk which worldwide insurance and reinsurance companies carry on their balance sheets (cf. Group of Thirty (2006)).

One prominent example of ILS, which enables (re)insurers to load extreme tail risks off their balance sheets, are so-called Catastrophe (CAT) Bonds, the yields of which depend on the incurrence of certain catastrophic events. They have been traded since the mid 1990s and present interesting investment possibilities as they are "low-beta" investments, i.e. their yields show a low correlation to financial markets, and thus increase diversification possibilities for investors (see Cox et al. (2000)). Moreover, they offer several potential advantages over alternative methods for insurers to deal with catastrophic risk (see Niehaus (2002)).

The market for CAT securities and the pricing of CAT bonds have been studied in various contributions (see e.g. Doherty (1997), Froot (2001) and Lee and Yu (2002), Young (2004), respectively). However, the amount of capital that has been raised within securitizations of catastrophic risk remains small (Cummins (2006)). One possible explanation is that these bonds may be expensive relative to conventional reinsurance since investors often charge a high risk premium on the bonds (cp. Bantwal and Kunreuther (1999), Froot (2001)).

A more recent capital market innovation are so-called CAT Mortality Bonds (henceforth CATM bonds). While most CAT bonds and other CAT derivatives depend on underlying loss indices, such as the Property Claim Services (PCS) loss index, CATM bonds are contingent on less artificial events: They are triggered by a catastrophic evolution of death rates of a certain population. Investors' demand for these securities seems to be very high, and in contrast to "conventional" CAT bonds, the number of deals has increased considerably over the last years: There were four major deals in 2006 with a total volume of more than $\$ 1.2$ Billion. Surprisingly, there have been very few contributions in the scientific literature on CATM bonds. 
Lin and Cox (2006) and Cox et al. (2006) develop an asset pricing model for mortality contingent securities in an incomplete market framework with jump processes. In particular, they propose a pricing method for CATM bonds. Modeling the underlying combined mortality index which triggers the bond by a geometric Brownian motion with a multiplicative jump component and distorting the resulting distributions by the so-called Wang transform (see Wang (2000) and Wang (2002)), they are able to explain market outcomes of existing mortality securitizations regarding investors' demand by analyzing the implied risk premiums. However, their pricing model remains static in the sense that no risk-adjusted dynamics are derived. Furthermore, they only focus on a single transaction, namely the first one, and do not provide an overview on the CATM market thus far; particularly, the stability of their findings regarding subsequent transactions is not examined. Also, modeling the index rather than the evolution of the underlying mortality may not be adequate as the index corresponds to one specific deal.

The present article aims to close this gap in the literature. Aside from providing a concise overview on the market history, we develop a risk assessment and pricing model which is based on stochastic modeling of the mortality intensity. Parametrizations of the proposed model based on three different calibration procedures are derived; we provide the resulting loss profiles as well as prices, and discuss the consequences of our findings.

The remainder of the text is organized as follows. After explaining the general structure of CATM bonds based on the so-called Tartan bond ${ }^{1}$ in Subsection 2.1, Section 2.2 provides an overview on the development of the CATM securitization market thus far. In particular, we present the characteristics of all deals until the end of 2006. In Subsection 3.1, we describe general modeling approaches focusing on those used in practice. After a short overview on continuous stochastic mortality models in general, we introduce our specification in 3.2 based on the so-called intensity based approach for credit risk modeling by Lando (1998). Three calibration procedures for this model are presented in Section 4: First, best estimate parametrizations based on historical data and viewpoints from the demographic literature are derived; the other two risk-adjusted parametrizations are extracted from market prices of term life insurance policies and past catastrophe mortality securitizations, respectively. Section 5 presents our results; we provide prices in terms of excess spread levels, loss probabilities, as well as expected losses corresponding to the parametrizations derived in Section 4 and compare our results to loss profiles provided by the issuers and market prices. Moreover, similarities to credit risky securities are pointed out. After a discussion of our findings, Section 6 concludes and provides an outlook on future research.

\section{Market Overview}

Securitization transactions are usually highly complex and involve several parties such as lawyers, rating agencies, trustees, etc. Providing a general overview on securitization transactions is far beyond the scope of this article (see e.g. Deacon (2006) or Jeffrey (2006) for an introduction to securitization and Asset Backed Securities (ABS) in general, or Cowley and Cummins (2005) for life insurance securitization). The basic idea is to isolate and pool cash flows that are linked to certain assets or liabilities, repackage them into cash flows which support certain related securities, and issue these securities to capital markets.

Within CATM securitizations, insurers and reinsurers transfer catastrophe mortality risk, which arises from a possible occurrence of, for example, severe pandemics

\footnotetext{
${ }^{1}$ Tartan Capital Ltd. Series 1 arranged by Goldman Sachs for the reinsurer Scottish Re Group Ltd. (Scottish Re), issued in May 2006.
} 


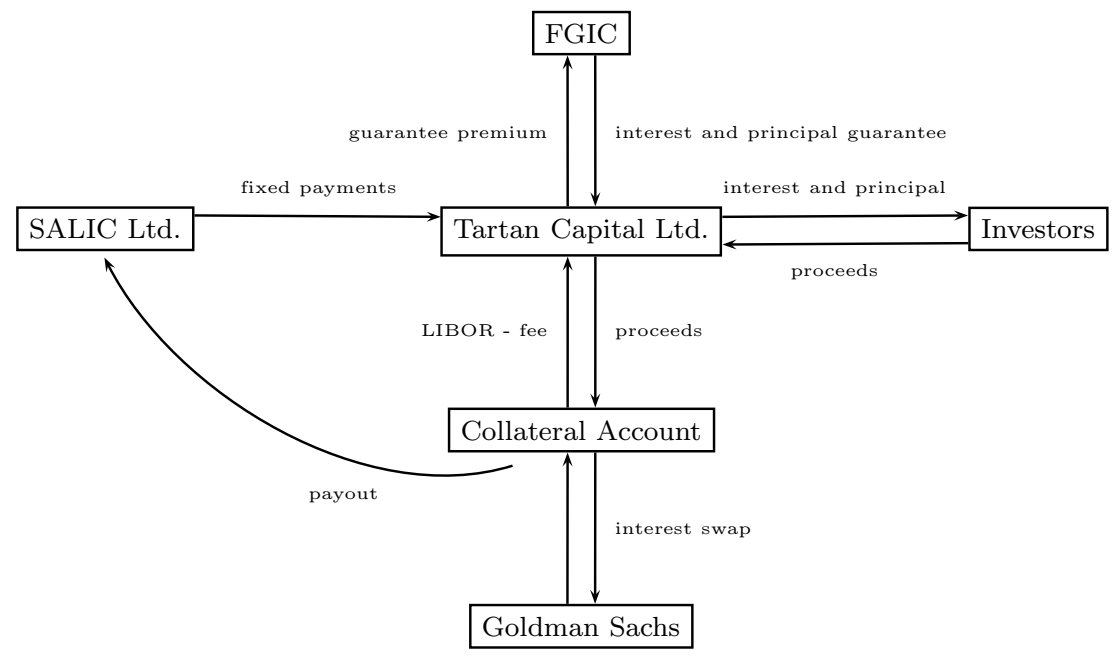

Figure 1: Tartan deal structure (Source: Linfoot (2007))

or catastrophic terrorist attacks, from their liability side to the capital market by means of CATM bonds. Traditionally, these risks were shared between insurers and reinsurers via reinsurance or retrocession. However, in contrast to these classical approaches, securitization avoids credit risk (see also Niehaus (2002)). Moreover, a traditional risk transfer may be more expensive as possible transaction partners usually already have this type of risk in their books, and thus their appetite for it is limited. Also, retrocession would require the disclosure of the own business to possible competitors.

Thus far, there have been five public transactions. While they differed in their coverage area, credit ratings, or spread levels, the basic structure is the same: A certain underlying mortality index based on the mortality experience in the coverage area is defined; if this index exceeds certain pre-specified levels, the bond is triggered, i.e. the investors start to loose their principal. As it is cumbersome to present the characteristics of all available CATM bonds at the same time, in Subsection 2.1, we detail out the structure of one representative example, namely the third of all five transactions: the Tartan transaction arranged by Goldman Sachs for the reinsurer Scottish Re (cp. Linfoot (2007)). In Subsection 2.2, we then compare this deal to the other transactions so far.

\subsection{Structure of a CATM bond based on the Tartan trans- action}

In Figure 1, the structure of the Tartan transaction is illustrated. SALIC ${ }^{2}$, a member of the Scottish Re Group Ltd., entered into a counter-party agreement with the special purpose vehicle Tartan Capital Ltd. (Tartan). Under this agreement Tartan is obligated to make payments to SALIC in case a certain index is triggered. In return, SALIC agreed to pay Tartan a certain fixed amount quarterly. In order to raise funds for the conditional payments to SALIC, Tartan issued and sold bonds to capital market investors; the proceeds were used to buy eligible securities which act as collateral. As these collateral assets could decrease in market value, Tartan went into a swap agreement with Goldman Sachs, who have also structured the deal:

\footnotetext{
${ }^{2}$ Scottish Annuity 85 Life Insurance Company (Cayman) Ltd.
} 
In return for the variable investment income from the collateral account, Goldman Sachs agreed to pay the 3 -month $\mathrm{LIBOR}^{3}$ minus a fee of 10 basis points (bps).

Tartan issued 2 series of 3 -year notes: a $\$ 75$ million $(\mathrm{mn}$ ) (Class A) and an $\$ 80$ $\mathrm{mn}$ (Class B) tranche with different risk exposures. In particular, within the Class A notes, both interest payments and the investors' principal are guaranteed by the monoline insurer Financial Guaranty Insurance Co. (FGIC). Therefore, the only risk that investors in the Class A notes have to face is credit risk. In return for the guarantee, FGIC received a premium from Tartan. Class B investors, on the other hand, are actually exposed to catastrophe mortality risk, i.e. they will lose interest and principal in case of a trigger event.

The bonds and thus the payment to SALIC are triggered if a well defined parametric index exceeds a certain level. This so-called combined mortality index (CMI) is contingent on the mortality experience of certain populations, and the objective is to design it such that the actual catastrophe mortality exposure of the protection buyer (SALIC / Scottish Re) is reflected as well as is possible. Within the Tartan transaction, this index is solely based on US population mortality. For each relevant point in time (calendar year) $t$, the mortality rates, i.e. the probabilities to decease within the following year for certain partitions of the whole population as reported from the Centers for Disease Control and Prevention (CDC), are weighted to determine a weighted population death rate $\hat{q}_{t}$ :

$$
\hat{q}_{t}=\sum_{\text {all } x} \omega_{x, m} \hat{q}_{m, x, t}+\omega_{x, f} \hat{q}_{f, x, t},
$$

where $\hat{q}_{m, x, t}$ and $\hat{q}_{f, x, t}$ are the mortality rates for age group $x$ in calendar year $t$ for males and females, respectively, and $\omega_{x, m} / \omega_{x, f}$ are the weights applied to the corresponding mortality rates. The weights for the Tartan transaction are displayed in Table 1 . Now, the actual index at time $t$, say $i_{t}$, is derived from the

\begin{tabular}{|l|cc|}
\hline \hline Age Groups $(x)$ & Age Weights: Male $\left(\omega_{x, m}\right)$ & Age Weights: Female $\left(\omega_{x, f}\right)$ \\
\hline $1-4$ & $0 \%$ & $0 \%$ \\
$5-14$ & $0.1 \%$ & $0.1 \%$ \\
$15-24$ & $0.4 \%$ & $0.4 \%$ \\
$25-34$ & $8.2 \%$ & $6.1 \%$ \\
$35-44$ & $26.0 \%$ & $12.7 \%$ \\
$45-54$ & $21.4 \%$ & $7.8 \%$ \\
$55-64$ & $9.8 \%$ & $2.7 \%$ \\
$65-74$ & $2.3 \%$ & $0.8 \%$ \\
$75-84$ & $0.6 \%$ & $0.4 \%$ \\
$84+$ & $0.1 \%$ & $0.1 \%$ \\
\hline Total & $68.8 \%$ & $31.2 \%$ \\
\hline \hline
\end{tabular}

Table 1: Gender and age weights for the Tartan transaction (Source: Linfoot (2007))

underlying weighted population death rates at times $t$ and $t-1$ as well as the weighted population death rates for the reference years 2004 and 2005, which are determined according to Equation (1), by the relationship

$$
i_{t}=\frac{\frac{1}{2}\left(\hat{q}_{t}+\hat{q}_{t-1}\right)}{\frac{1}{2}\left(\hat{q}_{2005}+\hat{q}_{2004}\right)} .
$$

Since the index relies on the experience of two consecutive years and since Tartan issued bonds with a three year tenor, there are only two dates at which the index is

\footnotetext{
${ }^{3}$ London Interbank Offered Rate.
} 
calculated and at which the principal may be reduced due to a potential catastrophic event: at the end of 2007 for the years 2006 and 2007, and at the end of 2008 for the years 2007 and 2008. In particular, this implies that investors cannot lose principal in the first two years. However, the data for the index calculation will usually not be available until awhile after the respective measurement dates. Therefore, Tartan has the possibility to extend the tenor of the notes up to a maximum of 30 months, but the securities cannot suffer any losses due to a possible event within the extension period, and investors will receive ongoing interest payments.

Furthermore, only if the index exceeds a certain level, the so-called trigger level or attachment point $a$, will investors lose principal. If the index exceeds the so-called exhaustion level or detachment point $d$, their complete principal will be lost. For index levels between the attachment and detachment points, the loss percentage of the principal at time $t=2007,2008, l_{t}$, is determined as follows:

$$
l_{t}=\min \left\{\max \left\{l_{t-1}, \frac{i_{t}-a}{d-a}\right\}, 100 \%\right\},
$$

where $l_{2006}:=0$. Coupons are only paid on the remaining principal. Table 2 provides the trigger and exhaustion levels as well as the interest on the Tartan notes.

\begin{tabular}{|l|cc|}
\hline \hline & Class A Notes & Class B Notes \\
\hline Tranche Size & $\$ 75 \mathrm{mn}$ & $\$ 80 \mathrm{mn}$ \\
Term & 3 years & 3 years \\
Trigger Level & $115 \%$ & $110 \%$ \\
Exhaustion Level & $120 \%$ & $115 \%$ \\
Coupon (bps) & LIBOR+19 & LIBOR+300 \\
Rating $^{4}$ & Aaa/AAA & Baa3/BBB \\
\hline \hline
\end{tabular}

Table 2: Program Summary of the notes issued by Tartan (Source: Linfoot (2007))

Interest on the notes is paid quarterly. It is worth mentioning that the spread levels are not fixed from the beginning of the marketing phase of the notes - they depend on investors' demand and market conditions.

\subsection{Market Development ${ }^{5}$}

As mentioned earlier in this section, Tartan was the third public catastrophe mortality transaction. Table 3 provides an overview on all such transactions so far.

Vita Capital Ltd. (Vita I) was the first CATM securitization transaction out of the $\$ 2$ billion (bn) multi-currency shelf program ${ }^{6}$ established by Swiss Reinsurance Company (Swiss Re). While the latter four deals are still ongoing, Vita I matured in the end of December 2006 and was not extended as there was no extreme mortality event during the risk period. In contrast to the Tartan deal, it only had one single tranche and the underlying index was based on the population from several countries rather than just one. ${ }^{7}$ When structuring the transaction, Swiss Re wanted to involve

\footnotetext{
${ }^{4}$ Rating at Issuance from Moody's Investors Service (Moody's) and Standard and Poor's (S\&P).

${ }^{5}$ Based on Logisch (2007).

${ }^{6}$ Shelf program means that not the total capacity is issued initially - some "sits on a shelf". Establishing a shelf program reduces costs for future transactions as the legal work, modeling, etc. are done for a relatively large amount. Moreover, it reduces the time from the decision to access the capital market and the closure of the deal enabling the issuer to quickly react when protection is needed or investors' appetite is large enough to absorb the extra issued bonds.

${ }^{7}$ In this case, the combined mortality index is defined as the weighted average over the indices from the individual countries determined according to Equation (2) with country weights as provided in Table 3.
} 
an American monoline insurer to "wrap" the bond, but due to regulatory issues no cooperation was established. Swiss Re managed the challenge of selling this new type of risk to the market, and the notes were placed successfully.

About 18 months after Vita I, again Swiss Re came to the market with their second transaction, Vita Capital II Ltd. (Vita II). In contrast to Vita I, Vita II has three tranches with different, decreasing seniorities due to decreasing trigger and exhaustion levels, but all of them are of a lower seniority than the single Vita I tranche. Despite this fact, the spread level within the first transaction exceeds the level of the Class B note from Vita II. This tight pricing was possible since the bond was over-subscribed indicating that within Vita I, investors had demanded a considerable novelty premium, i.e. an additional premium for the unknown asset class. However, Swiss Re did not seem to be surprised by this fact since they chose to issue the riskier and potentially more expensive tranches after the market had got acquainted with this type of security. As indicated in Table 3, S\&P upgraded all three Vita II classes by one notch in April 2006. According to Standard and Poor's (2006), this upgrade was mainly due to the availability of new mortality data showing mortality improvements, advances in vaccine research, and continuing work of governments regarding their pandemic preparedness plans.

As mentioned above, Tartan was the third CATM transaction, and so far the only one without involvement of Swiss Re. This first issue out of Scottish Re's $\$ 300 \mathrm{mn}$ shelf structure was also the first issue with a tranche wrapped by a monoline insurer. In comparison to the Vita II Class D tranche, the non-guaranteed Class B note is priced considerably higher even though both have the same trigger and exhaustion levels. This may be due to the fact that, in contrast to the deals before, Tartan is solely based on US mortality experience and, thus, there is no diversification effect among several populations. However, the more important reason was likely timing: During the marketing period of the transaction in the beginning of 2006, the international press had paid an increased attention to possible outbreaks of Avian Flu and to pandemics in general (see e.g. the Pandemic Theme Index provided by Conquest Investment Advisory AG).

Six months after Tartan, once the public discussions regarding pandemic fears had calmed down, the fourth series of CATM bonds were issued by OSIRIS Capital Plc (OSIRIS). Again, Swiss Re was involved as structurer and lead underwriter for the underlying EUR1.0bn shelf program but not as the protection buyer; the program has been structured as a securitization for the catastrophe mortality risk within the books of $A X A$ Cessions (AXA), a subcompany of the French $A X A$ group. Therefore, it is the first deal which involves a primary insurer, and for the first time, the underlying CMI is not dominated by US mortality experience. Aside from Swiss Re, IXIS Corporate and Investment Bank (IXIS) and Lehman Brothers Inc. (Lehman) were invited to act as co-underwriters. According to a press release from Swiss Re on 11/13/2006, investors' demand was very strong and all classes were oversubscribed. Euroweek (2006) even reports that all tranches were increased in size due to high investor demand and that all classes were priced well within the price guidance. However, even though the Baa2/BBB rated Class $\mathrm{C}$ tranche was priced tighter than the Baa3/BBB+ Class B note from Tartan, its price is still far from the level of the similar Vita II Class D Bond. This may be a consequence of increased investors' expectations after the Tartan transaction. The high demand was mainly due to the fact that in addition to specialized CAT bond investors, Swiss Re, IXIS, and Lehman also approached traditional ABS investors. Furthermore, with the $\mathrm{Ba} 1 / \mathrm{BB}+$ rated Class D note a non-investment grade tranche was offered within a CATM securitization for the first time. Thus, the deal has also drawn attention from hedge funds. All in all, $50 \%$ of the bonds were sold to asset managers, $20 \%$ to banks, and $25 \%$ to hedge funds (cp. International Financing Review (2006)). 


\begin{tabular}{|c|c|c|c|c|c|c|}
\hline Issued & $\begin{array}{l}\text { Vita Capital Ltd. } \\
\text { Nov. } 2003\end{array}$ & \multicolumn{3}{|c|}{$\begin{array}{c}\text { Vita Capital II Ltd. } \\
\text { Apr. } 2006\end{array}$} & \multicolumn{2}{|c|}{$\begin{array}{c}\text { Tartan Capital Ltd. } \\
\text { May } 2006\end{array}$} \\
\hline Class $^{8}$ & $\mathrm{~A}$ & $\mathrm{~B}$ & $\mathrm{C}$ & $\bar{D}$ & $\mathrm{~A}^{*}$ & $\mathrm{~B}$ \\
\hline Tranche Size & $\$ 400 \mathrm{mn}$ & $\$ 62 \mathrm{mn}$ & $\$ 200 \mathrm{mn}$ & $\$ 100 \mathrm{mn}$ & $\$ 75 \mathrm{mn}$ & $\$ 80 \mathrm{mn}$ \\
\hline Arranger & Swiss Re & & Swiss Re & & \multirow{2}{*}{\multicolumn{2}{|c|}{$\begin{array}{l}\text { Goldman Sachs } \\
\text { Scottish Re }\end{array}$}} \\
\hline Protection for & Swiss Re & & Swiss Re & & & \\
\hline Rating $^{9}$ & $\mathrm{~A} 3 / \mathrm{A}+$ & $\mathrm{Aa} 3 / \mathrm{A}^{* *}$ & $\mathrm{~A} 2 / \mathrm{BBB}+{ }^{* *}$ & Baa2/BBB-** & Aaa/AAA & Baa3/BBB+ \\
\hline Attachment Point & $130 \%$ & $120 \%$ & $115 \%$ & $110 \%$ & $115 \%$ & $110 \%$ \\
\hline Detachment Point & $150 \%$ & $125 \%$ & $120 \%$ & $115 \%$ & $120 \%$ & $115 \%$ \\
\hline Coupon (bps) & LIBOR+135 & LIBOR+90 & LIBOR +140 & $\mathrm{LIBOR}+190$ & LIBOR +19 & $\mathrm{LIBOR}+300$ \\
\hline $\begin{array}{l}\text { Expected Maturity } \\
\text { Covered Area }\end{array}$ & $\begin{array}{c}4 \text { years } \\
\text { US } 70 \%, \text { UK } 15 \%, \mathrm{~F} 7.5 \% \\
\text { I } 5 \%, \text { CH } 2.5 \%\end{array}$ & \multicolumn{3}{|c|}{$\begin{array}{c}\text { US } 62.5 \%, \text { UK } 17.5 \% \text {, } \\
\text { D } 7.5 \%, \text { J } 7.5 \%, \text { CAN } 5 \%\end{array}$} & \multicolumn{2}{|c|}{ US $100 \%$} \\
\hline
\end{tabular}

\begin{tabular}{|c|c|c|c|c|}
\hline Issued & \multicolumn{4}{|c|}{$\begin{array}{c}\text { Osiris Capital Plc. } \\
\text { Nov. } 2006\end{array}$} \\
\hline Class & $\mathrm{B}^{*}$ & $\mathrm{~B} 2$ & $\mathrm{C}$ & $\mathrm{D}$ \\
\hline Tranche Size & Euro $100 \mathrm{mn}$ & Euro $50 \mathrm{mn}$ & $\$ 150 \mathrm{mn}$ & $\$ 100 \mathrm{mn}$ \\
\hline Arranger & \multirow{2}{*}{\multicolumn{4}{|c|}{$\begin{array}{c}\text { Swiss Re } \\
\text { AXA }\end{array}$}} \\
\hline Protection for & & & & \\
\hline Rating & Aaa/AAA & $\mathrm{A} 3 / \mathrm{A}-$ & $\mathrm{Baa} 2 / \mathrm{BBB}$ & $\mathrm{Ba} 1 / \mathrm{BB}+$ \\
\hline Attachment Point & $114 \%$ & $114 \%$ & $110 \%$ & $106 \%$ \\
\hline Detachment Point & $119 \%$ & $119 \%$ & $114 \%$ & $110 \%$ \\
\hline Coupon (bps) & EURIBOR +20 & EURIBOR +120 & LIBOR +285 & LIBOR +500 \\
\hline Expected Maturity & 4 years & 4 years & 4 years & 4 years \\
\hline Covered Area & \multicolumn{4}{|c|}{ F $60 \%$, J $25 \%$, US $15 \%$} \\
\hline
\end{tabular}

\begin{tabular}{|c|c|c|c|c|c|c|c|c|c|}
\hline Issued & \multicolumn{9}{|c|}{$\begin{array}{l}\text { Vita Capital III Ltd. } \\
\text { Dec. } 2006\end{array}$} \\
\hline Class & A-IV* & $A-V^{*}$ & $\overline{A-V I^{*}}$ & A-VII & B-I & B-II & B-III & $\mathrm{BV}^{*}$ & $\mathrm{BVI}^{*}$ \\
\hline $\begin{array}{l}\text { Tranche Size } \\
\text { Arranger }\end{array}$ & $\$ 100 \mathrm{mn}$ & $\$ 100 \mathrm{mn}$ & Euro $55 \mathrm{mn}$ & Euro $100 \mathrm{mn}$ & $\begin{array}{l}\$ 90 \mathrm{mn} \\
\text { Swiss Re }\end{array}$ & $\$ 50 \mathrm{mn}$ & Euro $30 \mathrm{mn}$ & $\$ 50 \mathrm{mn}$ & Euro $55 \mathrm{mn}$ \\
\hline Protection for & & & & & Swiss Re & & & & \\
\hline Rating & Aaa/AAA & Aaa/AAA & Aaa/AAA & $\mathrm{Aa} 2 / \mathrm{AA}-$ & $\mathrm{A} 1 / \mathrm{A}$ & $\mathrm{A} 1 / \mathrm{A}$ & $\mathrm{A} 1 / \mathrm{A}$ & Aaa/AAA & Aaa/AAA \\
\hline Attachment Point & $125 \%$ & $125 \%$ & $125 \%$ & $125 \%$ & $120 \%$ & $120 \%$ & $120 \%$ & $120 \%$ & $120 \%$ \\
\hline Detachment Point & $145 \%$ & $145 \%$ & $145 \%$ & $145 \%$ & $125 \%$ & $125 \%$ & $125 \%$ & $125 \%$ & $125 \%$ \\
\hline Coupon (bps) & $\mathrm{LIBOR}+21$ & $\mathrm{LIBOR}+20$ & EURIBOR +21 & EURIBOR +80 & LIBOR +110 & LIBOR +112 & EURIBOR +110 & LIBOR +21 & EURIBOR +22 \\
\hline Expected Maturity & 4 years & 5 years & 4 years & $\begin{array}{l}5 \text { years } \\
\text { yS } 6250\end{array}$ & 4 years & 5 years & 4 years & 5 years & 4 years \\
\hline
\end{tabular}

Table 3: Deal Comparison (Source: New Issue Reports from S\&P and Moody's; Bloomberg data)

${ }^{8}$ The tranches marked with ${ }^{*}$ are guaranteed by monoline insurers.

${ }^{9}$ Rating at Issuance from Moody's / S\&P - the ratings marked with ${ }^{* *}$ were upgraded by S\&P. 
In late December of 2006, Vita Capital III Ltd. (Vita III) issued the third series of bonds out of Swiss Re's shelf program with the key objective to replace Vita I, which had expired in the same month. However, the total amount was increased in comparison to Vita I. All offered tranches are of a high seniority with all ratings above A, and they were priced similarly to the comparable OSIRIS notes. It is worth noting that five of the nine tranches are wrapped by three different monoline insurers.

In conclusion, it appears that in the relatively short history of the CATM market, the spectrum of investors has broadened considerably. While the initial transactions were mainly geared towards specialized CAT bond investors, more and more fixedincome and traditional ABS investors seem to be interested. This may be reasoned with the low correlation or the one-way relationship with debt capital and equity markets and the resulting diversification possibilities, but the attractive risk-return profile when comparing CATM bonds to similar rated Mortage Backed Securities (MBS) or Collateralized Debt Obligation (CDO) tranches certainly plays a role, too. ${ }^{10}$ For example, an anonymous investor explained that he is investing in CATM bonds because of the relative high spread margins and added: "If there will be one day such a severe world-wide pandemic that one of the bonds I bought will be triggered, there will be more important things to look after than an investment portfolio."

In order to estimate and analyze the risks within CATM bonds, investment managers started hiring actuaries to act as specialists on insurance risks. However, so far, the market participants mainly rely on the advice of so-called modeling firms. In the next section, after providing an overview of these consultants and their modeling approaches, we introduce a model which can be used to price and analyze extreme mortality risks.

\section{Modeling CATM bonds}

Aside from the arranger, SPV managers, rating agencies etc., so-called risk modeling firms play an important role in a catastrophe mortality securitization. They are appointed to calculate loss probabilities and expected losses for the different tranches of a transaction. These loss profiles are important as, usually, investors and rating agencies base their decisions on this data. Furthermore, they are in charge of calculating the combined mortality index; thus, they are also referred to as calculation agents. To date, the global acting actuarial consultant Milliman Inc. (Milliman) was hired as the calculation agent in all transactions so far. However, within the Vita III transaction, the US based company Risk Management Solutions (RMS) was also involved as an adviser for the monoline insurer Financial Security Assurance Inc. (FSA). The modeling approaches of Milliman and RMS differ considerably: While Miliman bases their analysis on an actuarial model, RMS uses an epidemiological approach. Although no mathematical details on their respective models can be presented as to our knowledge these are not published, an overview of their approaches based on the available information is provided in Subsection 3.1. In Subsection 3.2, we present our approach based on stochastic mortality modeling.

\footnotetext{
${ }^{10}$ The term one-way relationship means that adverse events in the financial market have no impact on the performance of a CATM bond, whereas a severe pandemic could affect the financial markets considerably.
} 


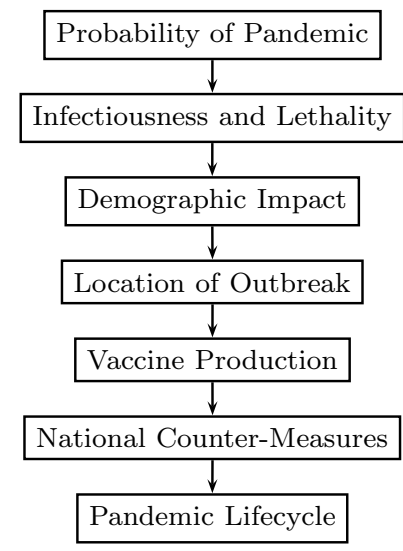

Figure 2: RMS Pandemic Influenza Model Framework (Source: Logisch (2007))

\subsection{General Modeling Approaches}

\section{RMS: An Epidemiological Approach ${ }^{11}$}

RMS reports that their catastrophe mortality model is based on epidemiological data and research rather than historical data, and that they were supported by world-wide experts in influenza research when developing their methodology. However, historical data is used to test the model. It is worth mentioning that their model does not include man made catastrophes such as terrorism acts.

Within their model, event tree techniques are applied to produce 1,890 probability weighted scenarios; Figure 2 shows the underlying event tree. Regarding the probability of an outbreak, RMS mentions that there have historically been an average of three pandemics per century. Thus, they assume an annual outbreak probability of $3-4 \%$. However, as industrialized livestock husbandry and other conditions fostering mutations of viruses have increased in recent decades, it is possible that the situation has worsened. Furthermore, the ongoing scientific debate of whether the risk of a pandemic is increased due to $\mathrm{H} 5 \mathrm{~N} 1$ prevalence in bird populations (the so-called Avian Flu) is another indication that the historical probability may be too low. Therefore, RMS advises using levels of $5 \%$ and $6.7 \%$ for stress-testing purposes.

The parameters determining infectiousness and lethality are fixed based on influenza research. In particular, they researched what proportion of the population is susceptible to a virus, which proportion will be affected, and what the corresponding recovery rates are. More precisely, the demographic impact of a virus is considered in their model as usually the very young and the elderly - those with a weaker immune system - are most affected by a virus. However, due to so-called "cytokine storms", i.e. potentially fatal immune reactions, a strong immune system may rather be a disadvantage than an advantage. For example, within the Spanish Flu from 1918, the most severe pandemic in the last century, a disproportionate amount of young adults had been killed, which is believed to be the consequence of cytokine storms. Furthermore, human deaths from H5N1 usually involve cytokine storms. ${ }^{12}$ The location of an outbreak is another important influence factor within the RMS model: Five world regions are being distinguished, and a regional as well as an interregional spread rate is modeled. For example, the underlying data includes maps

\footnotetext{
${ }^{11}$ Based on Logisch (2007), who reports a web presentation held by an RMS modeling expert in January 2007 as his primary source.

${ }^{12}$ From www.wikipedia.org, 02/09/2007.
} 


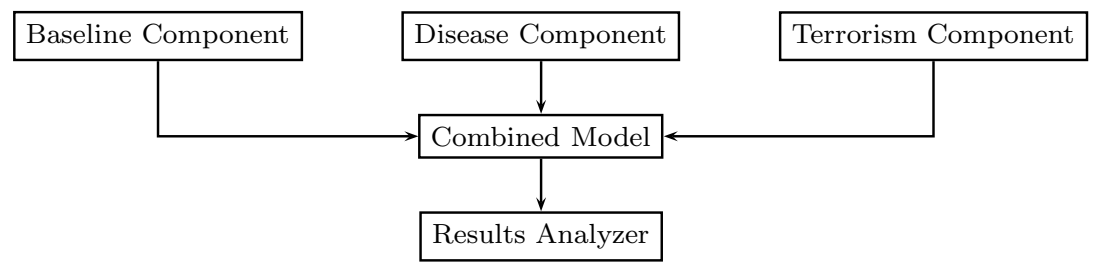

Figure 3: Milliman Model Overview (Source: Logisch (2007))

of the connections between international airports. According to RMS, their spread model yields results which are consistent with the models used by the US government, the UK government, and the World Health Organization. The location of the outbreak also determines the effectiveness of the country-specific emergency plans and countermeasures as well as vaccine production scenarios and their probabilities. RMS claims consistency of their model with all historic data. In their opinion, a severe pandemic would arise from the combination of a virulent and infectious virus, a high incidence of cytokine storms, a delayed vaccine, and a failed government response.

\section{Milliman: An Actuarial Approach ${ }^{13}$}

As noted above, Milliman's approach differs considerably from the one presented by RMS as Milliman models the future evolution of mortality with actuarial and statistical methods based on historical data. As shown in Figure 3, their framework consists of three basic components:

1. The Baseline Component models random fluctuations within annual mortality rates as long as no catastrophic event occurs. Using time-series models, they develop stochastic forecasts of the mortality evolution for every country covered in the combined mortality index which are applied to produce simulations of the weighted combined death rates (cp. Equation (1)).

2. The Disease Component captures the excess mortality due to a pandemic outbreak. The frequency and severity of an outbreak are modeled separately based on data from past pandemics. The same model is used for each relevant country, and it is assumed that pandemics occur simultaneously in these countries. Furthermore, they assume that these events are independent between calendar years.

3. The Terrorism Component produces simulations for mortality shocks arising from a terrorist attack based on a multi-level trinomial tree. In each level, there are three possible outcomes with different probabilities: "Failure", i.e. no deaths have occurred; "Success" of the attack, so a random number of deaths within a given range is assumed; or "Escalate", which means that the attack was more severe than attacks corresponding to the current level implying that the model jumps to the next level with a higher range of possible death counts. According to Linfoot (2007), the probabilities and ranges are calibrated to the terrorism model from the US State Department (for 19992003) and the National Counterterrorism Center (for 2004).

For each basic component 250,000 simulations are produced and combined to estimate annualized as well as cumulative expected losses and several loss probabilities

\footnotetext{
${ }^{13}$ Based on Linfoot (2007) and Logisch (2007).
} 
such as the probability that the trigger level of a certain tranche will be reached. Even though the terrorism component only has a marginal impact for these estimations, it was included into Milliman's model since the Vita II deal upon investors' requests. Furthermore, mortality shocks due to natural disasters are not modeled explicitly as "such events would not have resulted in a large enough number of deaths to cause a loss to any Class of the Notes [within the Tartan deal]."14

As noted above, Milliman's model was the primary basis for investors' and rating agencies' decisions within all CATM transactions thus far since Milliman was appointed as the risk modeling firm in each deal. The reason for this "monopolyposition" is probably that they were the first to have a model for catastrophe mortality risk which was accepted by the market. Since they are part of several ongoing shelf programs and since investors have become accustomed to seeing Milliman involved in the deals, it is very likely that they also will play a dominant role in the future. However, rumors in the market indicate that RMS is currently working on a transaction together with a large American insurer. As RMS reports substantially higher loss probabilities than Milliman, the consequences of an involvement of RMS in future transactions may considerably change current spread levels.

While RMS' idea of building an epidemiological model is interesting, from a mathematical point of view the massive amount of necessary parameters is problematic. For example, when including parameter uncertainty or when conducting sensitivity analyses, confidence bands for the loss probabilities become very large; therefore, we will follow Milliman's approach and present an actuarial model in the next subsection.

\subsection{Stochastic Mortality Modeling}

In order to analyze and eventually price CATM bonds, it is necessary to model the underlying combined mortality index. While Lin and Cox (2006) and Cox et al. (2006) directly model the weighted population death rate from Equation (1), we consider modeling the underlying cohort specific death rates. Understanding the index as a function of these death rates is, in our opinion, more adequate as the combined index corresponds to the specifications of a certain transaction, and therefore, a "direct" model of the index or the weighted population death rate will naturally be bound to the deal in view. Furthermore, we believe that the more basic approach coheres better with the structure of the problem and permits the consideration of more general transaction structures.

Several stochastic mortality models have been proposed in the literature - for an overview and a categorization of continuous mortality models, see Cairns et al. (2006). By replacing formerly constant parameters in the Gompertz mortality law ${ }^{15}$ with diffusion processes, Milevsky and Promislow (2001) were among the first to propose a stochastic hazard rate, which is also referred to as the (spot) force of mortality or the mortality intensity. Their idea has been extended and generalized in more recent contributions; in particular, the application of so-called affine processes, which are well known from interest rate and credit risk modeling, to stochastic mortality modeling has been brought forward by a number of authors due to their analytical tractability (see e.g. Biffis (2005), Dahl (2004), or Schrager (2006)).

We also make use of affine structures but focus on our particular model rather than providing a general overview on (affine) mortality models as this is beyond the scope of this article - we refer the interested reader to Biffis (2005). Furthermore, the

\footnotetext{
${ }^{14} \mathrm{cp}$. Linfoot (2007).

${ }^{15}$ Mortality Laws are certain parametric functions which are used to model human mortality; see Gompertz (1825) for a classical example or Bowers et al. (1997) for an overview on mortality laws.
} 
underlying force of mortality can be defined using several approaches. For example, Cairns et al. (2006) define the involved quantities via prices on a hypothetical exogenous mortality bond market, whereas Biffis et al. (2005) define the mortality intensity as the so-called hazard process of the time of death $\tau$. Here, we take a rather simple path and, similarly to Miltersen and Persson (2005), define the force of mortality based on the so-called intensity based approach from Lando (1998), which is well-known from credit risk modeling and arises as a special case from the approach presented in Biffis et al. (2005).

For the remainder of the article, we fix a time horizon $T^{*}$ and a filtered probability space $\left(\Omega, \mathcal{F}, \mathbf{F}=\left(\mathcal{F}_{t}\right)_{0 \leq t \leq T^{*}}, P\right)$ where $\mathbf{F}$ is assumed to satisfy the usual conditions, i.e. $P$-completeness and right continuity. Moreover, we fix a specific underlying population of individuals at inception, where each individual has a certain age denoted by $x_{0}$. Following Lando (1998), we further assume that an Fadapted, $d$-dimensional stochastic process $X=\left(X_{t}\right)_{0 \leq t \leq T^{*}}$, which is assumed to be right continuous with left limits (RCLL), as well as a positive, continuous function $\mu(\cdot, \cdot): \mathbb{R} \times \mathbb{R}^{d} \rightarrow \mathbb{R}_{+}$are given. We define the time of death $\tau_{x_{0}}$ of an individual $i$ of age $x_{0}$ as the first jump time of a Cox-process with intensity $\mu\left(x_{0}+t, X_{t}\right)$, i.e.

$$
\tau_{x_{0}}=\inf \left\{t: \int_{0}^{t} \mu\left(x_{0}+s, X_{s}\right) d s \geq E_{i}\right\},
$$

where $E_{i}$ is a unit-exponentially distributed random variable independent of $X$ and $E_{i}$ and $E_{j}$ are assumed to be independent for two different individuals $i \neq j$. As a consequence, the mortality intensity $\mu\left(x_{t}, t\right)$ of an $x_{t}$ year old person at time $t$ is, in our setup, a function of the age, $x_{t}$, and of the realization of $X$ at time $t$.

Considering only one single insured for now, let the subfiltrations $\mathbf{G}=\left(\mathcal{G}_{t}\right)_{0 \leq t \leq T^{*}}$ and $\mathbf{H}=\left(\mathcal{H}_{t}\right)_{0 \leq t \leq T^{*}}$ be given as the augmentations of the filtration generated by $\left(X_{t}\right)_{0 \leq t \leq T^{*}}$ and $\left(1_{\tau_{x_{0}} \leq t}\right)_{0 \leq t \leq T^{*}}$, respectively, that is - neglecting the null sets $\mathcal{G}_{t}=\sigma\left\{X_{s}, 0 \leq s \leq t\right\}, \mathcal{H}_{t}=\sigma\left\{1_{\tau_{x_{0}} \leq s}, 0 \leq s \leq t\right\}$, and we set $\mathcal{F}_{t}=\mathcal{G}_{t} \vee \mathcal{H}_{t}$.

From Equation (4), we can derive the $(T-t)$-year survival probability at time $t$ for an $x_{t}=x_{0}+t$ year old individual as

$$
{ }_{T-t} p_{x_{t}}^{(t)}:=E\left[1_{\tau_{x_{t}}>T} \mid \mathcal{G}_{t}\right]=E\left[\exp \left\{-\int_{t}^{T} \mu\left(x_{0}+s, X_{s}\right) d s\right\} \mid \mathcal{G}_{t}\right] .
$$

The results of Lando (1998) yield

$$
E\left[1_{\tau_{x_{0}}>T} \mid \mathcal{F}_{t}\right]=1_{\tau_{x_{0}}>t T-t} p_{x_{0}+t}^{(t)}
$$

and, particularly,

$$
E\left[1_{\tau_{x_{0}}>T}\right]=E\left[1_{\tau_{x_{0}}>T} \mid \mathcal{F}_{0}\right]={ }_{T} p_{x_{0}}^{(0)} .
$$

Based on this insight, we can now use the "classical actuarial toolbox" to model and price insurance products. Therefore, in addition to survival probabilities, we will also need death probabilities and, in particular, mortality rates: The $(T-t)$-year death probability at time $t$ for an $x_{t}=x_{0}+t$ year old individual is

$$
{ }_{T-t} q_{x_{t}}^{(t)}=1-{ }_{T-t} p_{x_{t}}^{(t)},
$$

and, as usual, we set $q_{x_{t}}^{(t)}={ }_{1} q_{x_{t}}^{(t)}$.

In order to specify a particular model, it is now sufficient to specify a certain RCLL process $X$ as well as a function $\mu(\cdot, \cdot)$. Dahl et al. (2006) propose mortality intensities $\mu\left(x_{t}, t\right), x_{t}=x_{0}+t$, of the form

$$
\mu\left(x_{t}, t\right)=\mu\left(x_{0}+t, X_{t}\right)=\mu^{0}\left(x_{0}+t\right) X_{t},
$$


where $X$ is a time in-homogeneous mean reverting square root diffusion process, the parameters of which can also depend on $x_{0}$, and the initial mortality intensities $\mu^{0}$ are of the Gompertz-Makeham form. However, as pointed out by Lin and Cox (2006), "mortality jumps" arising from catastrophes such as pandemics should be taken into account when modeling the evolution of mortality, particularly when focusing on catastrophic mortality risk. Therefore, we extend the model by Dahl et al. (2006) on the one hand by including mortality jumps in the form of a Gamma Ornstein-Uhlenbeck process $\Gamma$ but restrict it by choosing a time homogeneous mean reverting square root diffusion $Y$ and considering a simpler, pure Gompertz form for the initial mortality intensities. Thus, we choose $X=(Y, \Gamma)$ and propose mortality intensities of the form

$$
\mu\left(x_{t}, t\right):=\mu\left(x_{t}, Y_{t}, \Gamma_{t}\right)=Y_{t} e^{b x_{t}+c}+\Gamma_{t},
$$

where $b, c \in \mathbb{R}, Y$ evolves according to the stochastic differential equation (SDE)

$$
d Y_{t}=\alpha\left(\beta-Y_{t}\right) d t+\sigma \sqrt{Y_{t}} d W_{t}, \quad Y_{0}>0
$$

and $\Gamma$ is governed by the SDE

$$
d \Gamma_{t}=-\kappa \Gamma_{t} d t+d J_{t}, \quad \Gamma_{0}=0 .
$$

Here $\alpha, \beta, \sigma, \kappa$ are positive constants, $W$ is a standard one dimensional Brownian motion, and $J$ is a compound Poisson process with intensity $\lambda$ and positive, independent $\operatorname{Exp}(\zeta)$-distributed jumps. In particular, the proposed mortality intensities in (6) have an affine structure which enables us to provide a semi-analytical representation of the survival probabilities implied by them. We have

$$
\begin{aligned}
{ }_{T-t} p_{x_{0}+t}^{(t)}= & E\left[\exp \left\{-\int_{t}^{T} \mu\left(x_{0}+s, Y_{s}, \Gamma_{s}\right) d s\right\} \mid \mathcal{G}_{t}\right] \\
= & \exp \left\{u(T-t)+v(T-t) Y_{t} e^{b\left(x_{0}+t\right)+c}\right\} \\
& \exp \left\{-\frac{\Gamma_{t}}{\kappa}\left(1-e^{-\kappa(T-t)}\right)-\frac{\lambda(T-t)}{\zeta \kappa+1}\right\} \\
& \exp \left\{\frac{\lambda \zeta}{\zeta \kappa+1} \log \left[1+\frac{1}{\zeta \kappa}\left(1-e^{-\kappa(T-t)}\right)\right]\right\},
\end{aligned}
$$

where $u$ and $v$ satisfy the following Riccatti ordinary differential equations (ODEs)

$$
\begin{aligned}
& u^{\prime}(x)=\alpha \beta v(x) e^{b\left(x_{0}+T-x\right)+c}, u(0)=0, \\
& v^{\prime}(x)=-1-(\alpha-b) v(x)+\frac{1}{2} \sigma^{2} v^{2}(x) e^{b\left(x_{0}+T-x\right)+c}, v(0)=0 .
\end{aligned}
$$

See the Appendix for a derivation of Equation (8). Therefore, by solving the ODEs from Equation (9), we are able to compute survival probabilities and then, as mentioned earlier in this section, use the "classical actuarial toolbox" to price life insurance products.

However, so far we have fixed a specific probability measure $P$, even though different investors may have different opinions regarding the evolution of the future mortality implying different individual measures. Furthermore, prices for mortality contingent claims may include a risk premium for the inherent risk, which also leads to a different probability measure, the so-called pricing measure $Q$. Informally, by a change of measure, the structure of the model can be altered considerably: Not only could it affect the intensity process $\mu(\cdot, \cdot)$, but a market price for unsystematic or idiosyncratic risk may be included (see e.g. Biffis et al. (2005)). We will not consider loadings for the idiosyncratic component as we regard a whole population. 
However, when limiting the perspective to an insurer's portfolio, there may (or may not) be reasons for a premium for unsystematic risk (see Bauer and Russ (2006) for a discussion of this issue). Furthermore, when allowing for an (almost) arbitrary change of measure, the structure of the force of mortality could change tremendously under the "new" measure. Therefore, similarly to Dahl et al. (2006), we restrict ourselves to choices where the parameters of our setup can be changed but not the process' structure.

From a mathematical point of view, the analytical tractability of the presented model is a valuable and important feature that can be considered as a first advantage. A detailed discussion of our model choice in the context of actual mortality data is postponed to the next section where different calibration procedures are presented.

\section{Calibration of the Model}

When applying a financial model to determine risk-measures such as loss probabilities or expected losses, historical data is usually used to determine a parametrization which matches the past experience. However, as we are considering the evolution of mortality, not only past experiences but also the particular properties of mortality have to be taken into account. For example, mortality rates are positive. Furthermore, the projection of future death rates is not a purely statistical problem as demographic considerations should also be taken into account. Therefore, when calibrating our model to historic data in Subsection 4.1, we also incorporate demographical aspects.

When pricing contingent claims, on the other hand, it is usually not sufficient to rely on historical data as prices include premiums for the adopted risk. This risk premium generally cannot be determined endogenously, but it is implied by the market. Aside from catastrophe mortality securitization transactions, life insurance prices are subject to catastrophic mortality risk. Hence, a natural approach for determining a risk-adjusted parametrization for our model is to extract it from insurance prices. This indirect approach is presented in Subsection 4.2, whereas in Subsection 4.3 we calibrate the model using data directly from CATM securitization transactions.

\subsection{Backtesting the Model and Historical Parametrizations}

Most CATM transactions to date were primarily exposed to US mortality experience. Therefore, we limit our considerations to American mortality data. Furthermore, as within the combined mortality indices, male death rates are usually weighted more heavily than female death rates (see Table 1), we focus on male mortality experience; however, when assuming independence of the "regular" mortality evolution for the different cohorts and a simultaneous occurrence of pandemics in the relevant countries as in the Milliman model, including female or non-US mortality data is straigth-forward. Without these rather rigorous assumptions, i.e. when allowing for correlations and diversification effects across genders and populations, the calibration procedure will get more sophisticated as correlations need to be estimated and incorporated into the model. We leave the exploration of this issue for future work.

Our model consists of two independent components: A diffusion part which models the "regular" evolution of mortality, i.e. when no catastrophic event occurs, and a jump-part which models pandemics and other catastrophes. In what follows, we will refer to these two components as the Baseline Component and the Catastrophe 
Component, respectively. The independence of the two components allows us to carry out the calibration procedures separately.

\section{The Baseline Component}

We use annual, periodic male mortality data as available from the Human Mortality Database ${ }^{16}$ for our considerations. There, age specific death rates $m(x, t)$ are available for each year $t$ from 1959 until 2003. From these death rates, we derive the sample mortality intensities $\tilde{\mu}$ by the following approximation (for a proper definition and properties of death rates $m(x, t)$ see e.g. Bowers et al. (1997)):

$$
\begin{aligned}
\tilde{\mu}\left(x_{t}+0.5, t+0.5\right) & =\tilde{\mu}\left(x_{t}+0.5, t+0.5\right) \frac{\int_{0}^{1} l\left(x_{t}+s, t+s\right) d s}{\int_{0}^{1} l\left(x_{t}+s, t+s\right) d s} \\
& \approx \frac{\int_{0}^{1} l\left(x_{t}+s, t+s\right) \mu\left(x_{t}+s, t+s\right) d s}{\int_{0}^{1} l\left(x_{t}+s, t+s\right) d s} \\
& =m\left(x_{t}, t\right),
\end{aligned}
$$

where $l\left(x_{t}, t\right)$ denotes the exposures, i.e. the number of individuals within the relevant cohort of $x_{t}$-year aged males at time $t$. In order to simplify notation, we set the inception date $t=0$ to mid 1959, i.e. 30.06.1959, and thus we are given the spot intensities $\tilde{\mu}\left(x_{t}, t\right)$ for ages $x_{t} \in\{0.5,1.5, \ldots, 100.5\}$ in years $t=0$ (1959.5) through $t=44$ (2003.5). ${ }^{17}$ Within our model, neglecting the influence of the catastrophe component, the endogenous intensities $\mu\left(x_{t}, t\right)$ are of the form (see Equation (6))

$$
\mu\left(x_{t}, t\right)=Y_{t} e^{b x_{t}+c},
$$

where we conveniently set $Y_{0}=1$. Therefore, $\mu\left(x_{0}, 0\right)=e^{b x_{0}+c}$ is simply given by the Gompertz form, and the parameters $b$ as well as $c$ can be determined by an exponential regression on $\tilde{\mu}\left(x_{0}, 0\right)$; we obtain $b=0.08117916$ and $c=-8.7674591$. The comparison of the "actual" and model-endogenous mortality intensities is displayed in Figure 4. We find that the Gompertz approximation fits the data quite well. Particularly for years below 85, which are most relevant for the calculation of the combined mortality index (cf. Table 1), we only see slight deviations.

Similarly, Gompertz forms can be derived for all years $t$. However, within our model, $b$ and $c$ are constant over the years. By the relationship

$$
\mu\left(x_{t}, t\right)=e^{b x_{t}+c+\log \left\{Y_{t}\right\}} \Leftrightarrow \log \left\{\mu\left(x_{t}, t\right)\right\}-b x_{t}-c=\log \left\{Y_{t}\right\},
$$

we can estimate $\log \left\{Y_{t}\right\}$ as the mean of $\log \left\{\tilde{\mu}\left(x_{t}, t\right)\right\}-b x_{t}-c, x_{t}=0.5,1.5, \ldots$ and, hence, our model $\mu\left(x_{t}, t\right)$ by Equation (11). For example, in Figure 5, the actual-, the model-, and the Gompertz-mortality intensities for the years 36 (1995.5) and 43 (2002.5) are shown. Again, we can see that the model and the Gompertz intensities fit the data well in the relevant ages. However, the growth of the actual intensities is super-exponential for older ages, i.e. while the curve gets less steep for younger ages, it increases very fast for older ages. Furthermore, we find that the model and the Gompertz intensities are very close; for year 36 deviations are hardly noticeable, and for year 43 the deviations in the more relevant ages below 85 are also rather small.

\footnotetext{
${ }^{16}$ Human Mortality Database. University of California, Berkeley (USA), and Max Planck Institute for Demographic Research (Germany). Available at www.mortality.org or www.humanmortality.de (downloaded 11/03/2006 (1959-2002) and 04/11/2007 (2003)).

${ }^{17}$ Since the mortality index is only slightly affected by ages less than 30 and since there are structural deviations for very young ages and ages around 20 (so-called mortality humps), we only consider ages above 30 for the calibration.
} 


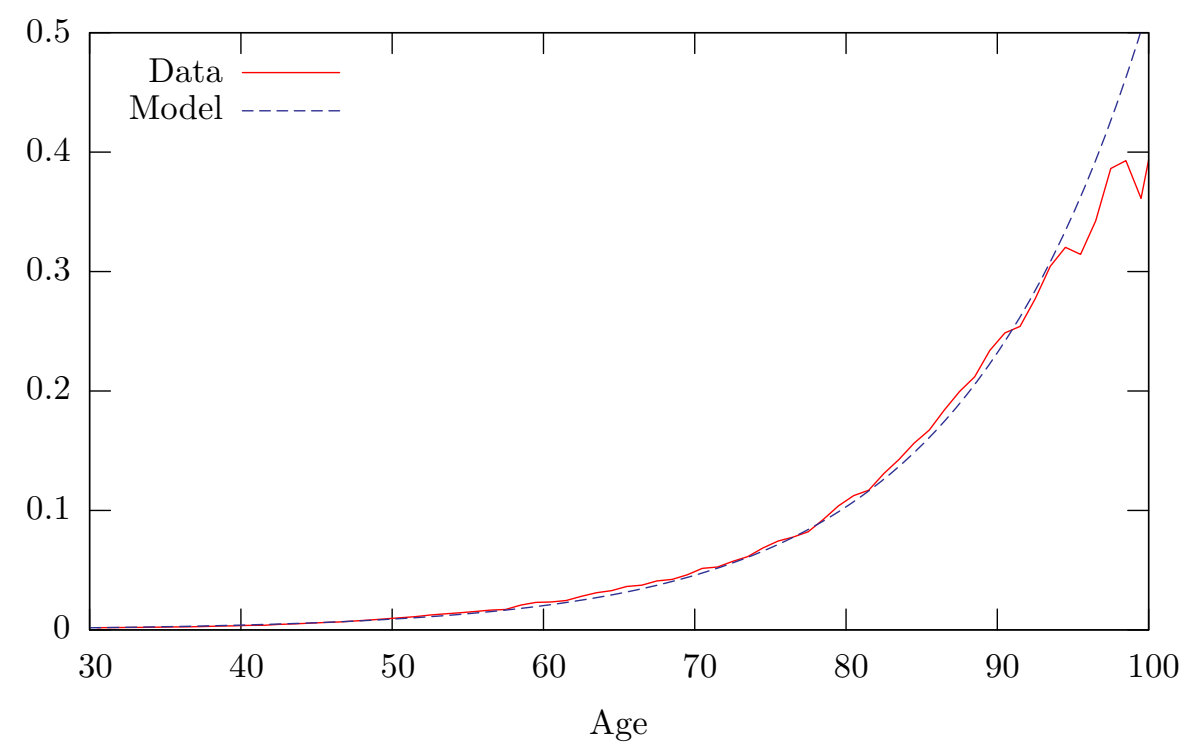

Figure 4: Mortality intensities 1959
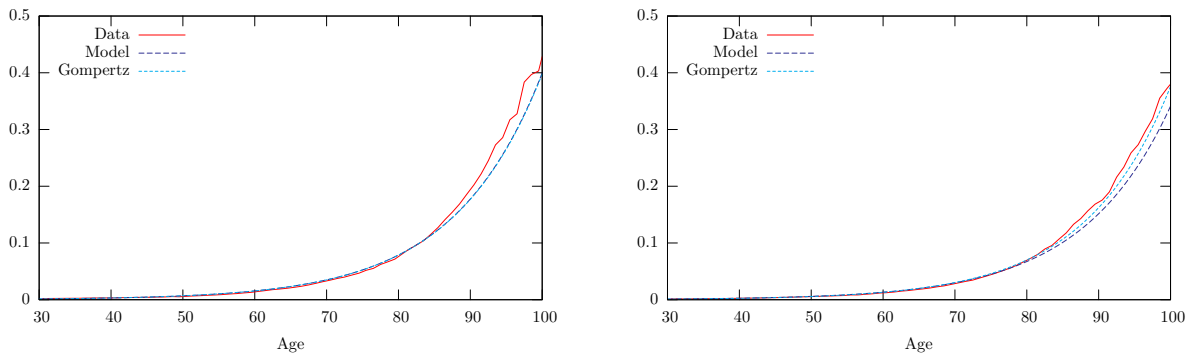

Figure 5: Mortality intensities for years 36 and 43 


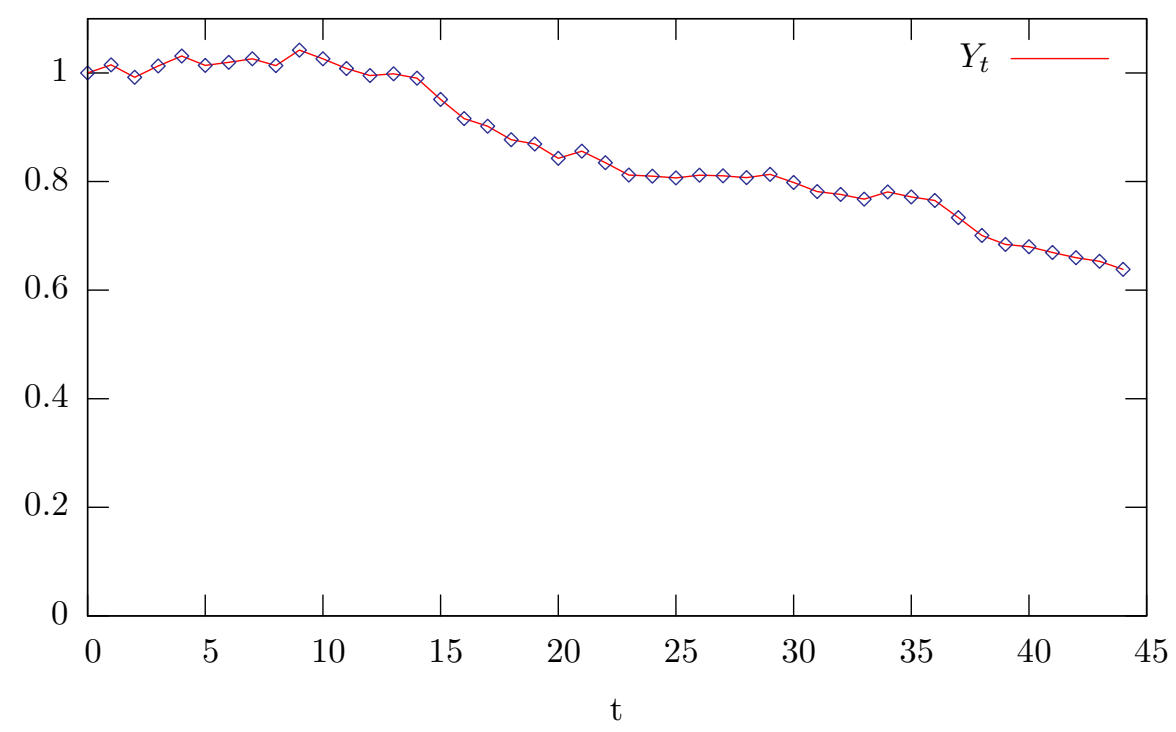

Figure 6: Time series of $Y$

By this procedure, we obtain a time series of the $Y_{t}$ for $t=0, \ldots, 43$, shown in Figure 6 , which can be used to estimate the parameters of the square root diffusion process $\alpha, \beta$, and $\sigma$ by estimators for the Cox-Ingersoll-Ross (CIR) interest rate model (see Cox et al. (1985)). However, there are two potential pitfalls.

Since we want to separate the influences of catastrophic events, such as pandemics, and "regular" deviations in mortality, we should not include peaks which are due to such catastrophic events. In the time between 1959 and 2003, there were two major occurrences: the so-called Hong-Kong Flu from 1968 and the so-called Russian Flu from 1977. The first is quite noticeable in our time series as there is a peak at the data point $t=9$ (1968.5). In order to disregard these influences, we smooth the time series by linearly interpolating these data points by the surrounding ones and taking the interpolated value instead of the recorded one whenever the interpolated value is lower.

We cannot observe a mean reversion trend in the time series. This fact could be interpreted as a problem with the specification or the general structure of the model, i.e. that a mean reverting process does not present a suitable choice. In fact, the question whether mean reverting processes are adequate for describing the evolution of mortality has been raised before in the literature (see e.g. Luciano and Vigna (2005)).

In this regard, Oeppen and Vaupel (2002) show that the average life expectancy in the country with the current highest life expectancy has increased almost strictly linearly at a little under three months per year over the last 160 years. Even though the annual records were set by only two nations since 1975, namely Iceland and Japan, the observations for other industrialized countries are quite similar. Therefore, they do not believe in a barrier for the life expectancy, which would be implied by a positive mean reversion level. Furthermore, they note that there is a long history of conjectured barriers, which were all broken only shortly after their publication.

A continuing linear trend of life expectancies may even require mortality intensities to decrease faster than observed over recent years: Keyfitz (1985) shows that a reduction of mortality intensities across all ages of $\delta \%$ would imply an increase of 
the life expectancy by $\delta H(t) \%$, where $H(t)$ is the so-called demographic entropy at time $t$, and investigations by Olivieri (2001) and Pitacco (2004) indicate that the demographic entropy is decreasing towards zero. ${ }^{18}$

However, Olshansky et al. (2001) believe that a faster decrease of death rates is very improbable. They argue that, on the one hand, death rates for young ages are almost at their minimum value. ${ }^{19}$ Therefore, reductions in high ages would need to account for increasing life expectancies, which eventually would lead to a so-called negligible senescence, i.e. mortality rates would remain constant for all attainable ages and aging would not reduce survival probabilities. According to the authors, this contradicts basic biological ideas and is thus not likely, if not impossible. On the other hand, they note that social-political and economical reasons indicate that life expectancies will not increase continuously.

We do not want to join this discussion (see Kristen (2007) for more details), but it appears that there is no general agreement among demographers regarding the future evolution of life expectancies.

Our model does not allow for systematically faster decreasing mortality rates in the future when choosing a positive mean reversion level $\beta$ and speed of mean reversion $\alpha .{ }^{20}$ Furthermore, when applying the maximum likelihood estimators from Walter (1996), these result in a negative speed of mean reversion $\alpha$ and a mean reversion level $\beta$ greater than 1, which indicates a strictly negative drift term of the process displayed in Figure 6. We may solve this problem by allowing for a deterministic function $\beta_{t}$ as the mean reversion level in our specification of $Y$ (see Equation (7)) rather than a constant. For example, $\beta$ could be replaced by

$$
\beta_{t}=e^{-\beta_{1} t}+\beta_{2}, \quad \beta_{1}, \beta_{2}>0 .
$$

A deterministic mean reversion level $\beta_{t}$ would not affect the analytical tractability of the proposed model. However, the number of model parameters would increase complicating calibration procedures. This particularly seems problematic in view of the limited data availability of only 44 data points. Moreover, even with a constant mean reversion level, our specification permits modeling all presented demographic viewpoints by choosing a coherent value of $\beta$. Therefore, we restrict ourselves to constant mean reversion levels, but we intend to further explore the possibility of choosing a deterministic mean reversion level in future work.

Instead of following only one particular demographic opinion, we will consider three parametrizations $\mathrm{P} 1, \mathrm{P} 2$, and $\mathrm{P} 3$, where the first $(\beta=0.6)$ and the third $(\beta=0)$ correspond to the "extreme" points of view from Olshansky et al. (2001) and Oeppen and Vaupel (2002), respectively, whereas P2 $(\beta=0.4)$ is a choice in the middle. Table 4 displays the resulting parametrizations. The speed of mean reversion $\alpha$ and

\begin{tabular}{|c|ccc|}
\hline \hline & $\beta$ & $\alpha$ & $\sigma$ \\
\hline P1 & 0.6 & 0.0325 & 0.01647 \\
P2 & 0.4 & 0.01829 & 0.01582 \\
P3 & 0.0 & 0.00976 & 0.01552 \\
\hline \hline
\end{tabular}

Table 4: Parametrizations of $Y$

the volatility $\sigma$ have been determined by the estimators presented in the Appendix.

\footnotetext{
${ }^{18}$ In the literature, this development is usually referred to as rectangularization as the shape of the mortality intensity curve gets more "rectangular", i.e. deaths occurrence is concentrated around a certain modal age (see also Figure 5).

${ }^{19}$ Demographers generally assume a minimal level of death rates for all age groups, which is motivated by the natural occurrence of accidents etc.

${ }^{20}$ This is due to the fact that in our specification, the drift of $Y$ decreases as it gets closer to the mean reversion level.
} 


\section{The Catastrophe Component}

In Lin and Cox (2006), catastrophic events are modeled as multiplicative shocks on the combined mortality index. While this approach could be reasoned by the idea that due to weaker immune systems the elderly population may be most affected by a possible pandemic in absolute terms, cytokine storms, which are believed to have been present during severe pandemics such as the Spanish Flu, may lead to a disproportionate amount of deaths in younger ages (cp. Section 3.1). Furthermore, man made catastrophic events such as severe terror attacks are not likely to affect older aged individuals more than younger individuals. Therefore, it seems to be appropriate to model catastrophic events as additive shocks, and we included the catastrophe component as an additive jump part to the mortality intensities (see Equation (6)).

For the calibration of the catastrophe component, we rely on the data from Linfoot (2007), where the frequency and severity of historical occurrences of infectious disease epidemics based on U.S. population experience are provided. This choice is motivated by the fact that this data was also used by the risk modeling firm Milliman within the Tartan transaction, and we want to keep our findings comparable; discussing the quality or adequacy of the data is beyond the scope of this article.

Linfoot (2007) reports an annual frequency of $7.4 \%$ (31 occurrences in the past 420 years) and severities for 5 (6) specific occurrences (model points) ${ }^{21}$. Therefore, we set the jump intensity of the compound Poisson process within the catastrophe component to $\lambda=7.4 \%$. Percentiles for the likelihood of each model point are derived by considering the number of equal or worse pandemics in relation to all occurrences. For example, the Spanish Flu is taken as a 1 in 420 years event, and given the annual frequency of $7.4 \%$, this yields the $\frac{1}{420} \frac{1}{0.074} \approx 0.032$ percentile.

The impact on the force of mortality which a catastrophic event implies $\tau$ years after its first occurrence in our model is given by $\Delta e^{-\kappa \tau}$ with $\Delta$ denoting the initial "jump". We assume that the event basically affects the mortality for one year only, i.e. that the influence after a year has decreased to only $1 \%$ of the initial impact, and thus set

$$
\Delta e^{-\kappa \cdot 1}=0.01 \Delta \Longleftrightarrow \kappa=4.6052 .
$$

For fitting the exponential distribution of $\Delta$, the given severities for the five model points are used. However, there the (multiplicative) excess mortality as a percentage of the mortality probability over all ages is provided. In order to use them for our considerations, they need to be "translated" to additive excess mortality intensities. As the population is not homogeneous across all ages, it is not sufficient to compute excess mortality intensities resulting from distorted mortality rates for every age and take the mean, but they have to be properly weighted: For the male population death rate at time $t$, we have

$$
\tilde{q}_{t}^{m}=\sum_{\text {all ages } x} \tilde{\omega}_{x}^{(t)} \hat{q}_{m, x, t}
$$

where $\tilde{\omega}_{x}^{(t)}$ are the male population weights, and $\hat{q}_{m, x, t}$ is the mortality rate for an $x$-year old male at time $t .^{22}$ Hence, if $e$ denotes the excess mortality rate, the corresponding initial impact $\Delta$ of the catastrophic occurrence can be approximated

\footnotetext{
${ }^{21} 1918$ (Spanish Flu), 1957 (Asian Flu), 1968 (Hongkong Flu), 1977 (Russian Flu) and 2003 (SARS). We omitted one data point called "Adjusted 1918-20" as it was fixed at the 0.0 Percentile Level.

${ }^{22}$ Note that the weights $\tilde{\omega}_{x}^{(t)}$ are the actual population weights and, thus, do not coincide with the deal-specific weights from Equation (1).
} 
by

$$
\begin{aligned}
(1+e) \tilde{q}_{t}^{m} & =(1+e) \sum_{\text {all ages } x} \tilde{\omega}_{x}^{(t)} \hat{q}_{m, x, t} \\
& \stackrel{!}{=} \sum_{\text {all ages } x} \tilde{\omega}_{x}^{(t)}\left(1-e^{-\int_{0}^{1} \mu(x+s, t+s)+\Delta e^{-\kappa s} d s}\right) \\
\Rightarrow \frac{\Delta}{\kappa}\left(1-e^{-\kappa}\right) & =\log \left\{\frac{1-\tilde{q}_{t}^{m}}{1-(1+e) \tilde{q}_{t}^{m}}\right\} .
\end{aligned}
$$

Therefore, given $e$, we still need to fix mortality rates and population weights to determine $\Delta$. We use mortality rates as implied by the Gompertz forms and population weights as provided by the U.S. Census Bureau ${ }^{23}$ for years 1959 and 2003, respectively. By matching the quantiles of an exponential distribution for the model points, we arrive at the parameters displayed in Table 5 . Aside from an exponential

\begin{tabular}{|lc|c|c|}
\hline \hline & & 1959 & 2003 \\
\hline $\operatorname{Exp}(\zeta)$ Distribution & $\zeta$ & 317.77 & 357.31 \\
& mean $=\frac{1}{\zeta}$ & 0.003147 & 0.002799 \\
\hline Gamma $\left(g_{1}, g\right)$ Distribution & $g_{1}$ & 0.57298 & 0.57293 \\
& $g_{2}$ & 182.08 & 204.71 \\
\hline \hline
\end{tabular}

Table 5: Parametrizations for the jump size distribution

distribution for the jumps, we also fit a Gamma distribution. Keeping the mean at the same level as for the exponential distribution in order to obtain comparable results and choosing the parameters that provide the best match with the model points in a (weighted) Least Squares sense, we arrive at the parameters displayed in Table 5.

We find that for a different demographic structure and different mortality rates, the calibrated parameters are quite different: For the exponential distribution, the mean is reduced by approximately $11 \%$ from the 1959 to the 2003 estimates. However, it is not clear which parametrization is more adequate; on the one hand, the 2003 demographic structure resembles the actual structure today, but on the other hand, the 1959 demographic structure may better cohere with the demographic structure when (severe) pandemics occurred. In order to keep our presentation concise, if not stated otherwise, we rely on the parametrization for 2003 and exponentially distributed jumps.

It is worth noting that due to the data used, the catastrophic component is subject to a high parameter uncertainty. For example, when only considering pandemic data from the last century, we may still use the data points from Linfoot (2007), but the resulting quantiles change considerably: When proceeding analogously, for the Spanish Flu we would obtain the $\frac{1}{100} \frac{1}{0.074} \approx 0.135>0.032$ percentile. Moreover, the given annual frequency of $7.4 \%$ is rather high in comparison to the frequency proposed by RMS $(3-4 \%)$ or by Cox et al. $(2006)(\approx 3.3 \%)$. Thus, it is necessary to conduct detailed sensitivity analyses for the catastrophe component. Furthermore, man made catastrophes are not explicitly modeled because we assume that the structural affect on mortality rates is very similar to a pandemic occurrence. However, as past events had a negligible effect on general population mortality, they are not considered in our calibration procedure.

\footnotetext{
${ }^{23}$ U.S. Census Bureau. National population estimates (male), www.census.gov/popest (downloaded 04/19/2007).
} 


\subsection{Risk-Adjusted Calibration Based on Insurance Prices}

If life insurers knew the future evolution of mortality, mortality risk management would be simple: With an increasing number of insured, the risk per sold policy would decrease to zero by the Law of Large Numbers. However, aside from this diversifiable, "unsystematic" mortality risk, insurance companies are also exposed to "systematic" mortality risk as the future evolution of aggregate mortality actually is not deterministic. Catastrophe mortality transactions allow insurers to transfer a part of this systematic risk, namely the part due to the possible occurrence of a catastrophic event, to the capital market. These transactions or, more specifically, the excess spreads are financed by the insurer and, hence, eventually by insurance premiums. This means that these premiums must account for catastrophic mortality risk. Thus, it is a natural idea to use life insurance prices to derive a risk adjusted parametrization for the mortality intensity process.

Similar ideas have been proposed for pricing longevity bonds (see Lin and Cox (2005)) and classical catastrophe derivatives (see Muermann (2003)). As pointed out by Bauer and Russ (2006), some conditions need to be satisfied regarding insurance prices and the insurance market in order to derive the risk premium for systematic mortality risk from insurance prices. In particular, insurance prices should not include a loading for unsystematic mortality risk, ${ }^{24}$ and the insurance market should be free of arbitrage. We refer to their article for a discussion of these assumptions.

The basic approach is straight-forward: Using our model, we compute prices for term life insurance policies and derive parameters such that the model-endogenous prices match market quotes optimally in the least squares sense. We consider term life insurance contracts with different maturities $T_{i}$ and individuals of different ages $x_{0}$ at inception, ${ }^{25}$ who get paid a fixed death benefit $\mathrm{D}$ against fixed, monthly premiums $\mathrm{P}$. Hence, the expected discounted value of the benefits $\mathrm{EB}_{T_{i}, x_{0}}(\mathrm{D})$ and the premiums $\mathrm{PB}_{T_{i}, x_{0}}(\mathrm{P})$ are given by the following equations:

$$
\begin{aligned}
\mathrm{EB}_{T_{i}, x_{0}}(\mathrm{D}) & =E\left[\sum_{t=0}^{T_{i} 12-1} \mathrm{D} e^{-r \frac{t+1}{12}} 1_{\frac{t}{12}<\tau_{x_{0}} \leq \frac{t+1}{12}}\right] \\
& =\sum_{t=0}^{T_{i} 12-1} \mathrm{D} e^{-r \frac{t+1}{12}}\left(E\left[1_{\tau_{x_{0}}>\frac{t}{12}}\right]-E\left[1_{\tau_{x_{0}}>\frac{t+1}{12}}\right]\right) \\
& =\mathrm{D} \sum_{t=0}^{T_{i} 12-1} e^{-r \frac{t+1}{12}}\left(\frac{t}{12} p_{x_{0}}^{(0)}-{ }_{\frac{t+1}{12}} p_{x_{0}}^{(0)}\right) \\
\mathrm{PB}_{T_{i}, x_{0}}(\mathrm{P}) & =E\left[\sum_{t=0}^{T_{i} 12-1} \mathrm{P} e^{-r \frac{t}{12}} 1_{\tau_{x_{0}}>\frac{t}{12}}\right] \\
& =\mathrm{P} \sum_{t=0}^{T_{i} 12-1} e^{-r \frac{t}{12}} \frac{t}{12} p_{x_{0}}^{(0)},
\end{aligned}
$$

where $r$ denotes the (constant) rate of interest. For the remainder of the article, we assume a constant short rate of $r=5.05 \%$, which corresponds to the 1 year U.S. treasury constant maturity date rate from $01 / 11 / 2007 .{ }^{26}$ For a given set of

\footnotetext{
${ }^{24}$ Technically, this means that the change of measure implied by including a risk premium should not affect the idiosyncratic jump component (cp. Section 3.2).

${ }^{25}$ Note that in contrast to the last subsection, for the remainder of the text we set the inception date 0 to January 1st, 2006.

${ }^{26}$ FRED (Federal Reserve Economic Data) provided by the St. Louis Federal Reserve Bank (www.research.stlouisfed.org). As the yield curve has only a mild downward slope, we consider the one year treasury yield as a fair proxy for our considerations.
} 
parameters, the quantities ${ }_{t} p_{x_{0}}^{(0)}$ can be conveniently calculated using Equation (8). By the actuarial principle of equivalence (see e.g. Bowers et al. (1997)), we then obtain the model-endogenous premiums

$$
\hat{\mathrm{P}}_{T_{i}, x_{0}}=\frac{\mathrm{EB}_{T_{i}, x_{0}}(\mathrm{D})}{\mathrm{PB}_{T_{i}, x_{0}}(1)},
$$

which depend on the parameter choice. Hence, the task is to find parameters such that the target function

$$
\sum_{T_{i}, x_{0}}\left(\hat{\mathrm{P}}_{T_{i}, x_{0}}-\mathrm{P}_{T_{i}, x_{0}}\right)^{2} \rightarrow \min
$$

is minimized, where $\mathrm{P}_{T_{i}, x_{0}}$ are the actual market quotes. Our data set contains prices as provided by Quickquote.com for male Californians ${ }^{27}$ with ages ranging from 25 to 55 and maturities from 10 up to 30 years. This calibration routine as well as all other numerical calculations are implemented in $C++$ using routines from the GNU Scientific Library ${ }^{28}$ (GSL); in particular, we make use of an ODE solver provided in the GSL based on the Runge-Kutta method.

But again, there are two potential pitfalls:

1. Death rates for the population of insured and the general population differ considerably, but CATM bond prices depend on population mortalities, whereas insurance prices depend on insured mortalities. Hence, assuming equality would not be adequate. However, this is not the case for mortality improvements: Even though mortality improvements were somewhat higher for the population of insured in comparison to the general population, the deviation is rather small. For example, for the incorporation of mortality trends into the Valuation Basic Table 2001 (VBT 2001), the American Academy of Actuaries reports that they relied on the improvements for the general population (cf. American Academy of Actuaries (2002)). Therefore, we assume that mortality improvements, which in our model are governed by the process $Y$, are alike for the population of insured and the general population. Furthermore, it seems to be reasonable that the exposure to catastrophes do not differ between the two populations, and therefore we also assume that the process $\Gamma$ is the same for both populations. Thus, it is sufficient to apply different parametrizations for the initial mortality intensity $e^{b x_{0}+c}$ to the different populations.

2. Insurance prices include adjustments for selection effects, which e.g. arise due to mandatory health examinations before underwriting the policies. Hence, when determining insurance prices based on mortality rates without selection effects included, the resulting prices are higher than corresponding prices based on selection tables. Since our model does not take selection effects into account, resulting parametrizations tend to underestimate risk and respective premiums included in insurance prices, but yield a lower bound.

In order to carry out the optimization algorithm, we still need to fix the initial mortality intensity, i.e. we need to find appropriate parameters $b$ and $c$. We use the VBT 2001, where period (spot) mortality rates $q_{x}^{(2001)}$ are provided. By a similar approximation to the one used in Equation (10), we can derive mortality intensities via the relationship

$$
\begin{aligned}
q_{x_{t}}^{(2001)} & =1-e^{-\int_{0}^{1} \tilde{\mu}\left(x_{t}+s, 2001+s\right) d s} \\
& \approx 1-e^{-\tilde{\mu}\left(x_{t}+0.5,2001+0.5\right)} .
\end{aligned}
$$

\footnotetext{
${ }^{27}$ Non-Smoking, Standard-Plus, monthly premium payments, $\$ 100,000$ coverage.

${ }^{28}$ See www.gnu.org for detailed information.
} 


\begin{tabular}{|ccccccc|}
\hline \hline \multicolumn{2}{l}{ Fixed Parameters } & & & & \\
\multicolumn{1}{l}{$c$} & & & & \\
& $c$ & $Y_{0}$ & $\Gamma_{0}$ & & \\
\hline 0.09697472 & -10.16051938 & 0.630240494 & 0 & & \\
& & & & & \\
Calibrated Parameters & & & & \\
$\alpha$ & $\beta$ & $\sigma$ & $\kappa$ & $1 / \zeta$ & $\lambda$ \\
\hline 0.1528 & 0.2234 & 0.0003393 & $6.370 \mathrm{e}-16$ & 0.002860 & 0.06441 \\
& & & & & \\
\hline
\end{tabular}

Table 6: Parameter calibration based on insurance prices

However, mortality intensities for $t=0$, i.e. $2006+0$ rather than for $2001+0.5$, are needed. We approximate them using the same methodology as was implemented for the derivation of the VBT 2001 (see American Academy of Actuaries (2002)); here, the 2001 data was extrapolated from 1990-1995 Basic Tables derived by the Society of Actuaries' (SOA) Individual Experience Committee. By applying the same trends, we further extrapolate the 2001 data to 2005.5 and derive mortality intensities $\tilde{\mu}(x, 2006)$ by relationship (13), and hence, parameter $b=0.09697$ and parameter $c=-10.62217$ by exponential regression. Of course, when choosing the starting value of the continuous part $Y_{0}$ different than $1, c$ has to be adjusted accordingly.

In the calibration procedure, we encounter numerical instabilities due to local minima of the target function. We solve this problem by considering a large set of different starting values for the optimization algorithm and choose the parameters which imply the minimum value for the target function within the considered set. The resulting parameter estimates are displayed in Table $6 .^{29}$

We find that the parametrization for the baseline component significantly differs from the results in the foregoing section. This may be a sign that the death rates derived from the VBT 2001 do not present a very good match to the death rates underlying the population of insured in view: The high mean reversion speed as well as the low volatility imply that the spot mortality intensities decrease very rapidly to levels close to $\beta e^{b x+c}$. For the catastrophe component, on the other hand, the resulting expected jump size $\frac{1}{\zeta}$ (jump intensity $\lambda$ ) is only slightly increased (decreased) compared to the historical value, but the impact over time, which the occurrence of a pandemic has on mortality rates, is substantially higher since $\kappa$ is almost zero.

These outcomes indicate that we have to be careful with definite conclusions since we did not include selection effects and since we can not be sure whether the initial mortality data from the VBT 2001 presents a good approximation for the population of insured in view. Considering other mortality tables or a different set of insurance quotes may yield more reliable results. For example, using prices from continuing options for existing term life insurance contracts, where selection effects usually are less dominant, may be worthwhile.

\footnotetext{
${ }^{29} Y_{0}$ was fixed at the same level as for the 2006 population mortality (cp. Section 4.3), and $c$ was adjusted accordingly.
} 


\subsection{Parameters Implied by Market Prices}

When pricing credit derivatives, the parameters of a given model are usually calibrated to market prices of certain securities such as Credit Default Swaps (CDSs) or CDOs with different maturities. Similarly, we may also parametrize our model based on prices of different CATM transactions or tranches within one transaction. In order to derive such an implied parametrization, but also to eventually analyze and price a transaction given some parameters, it is necessary to model the combined mortality index contingent on the basic quantities within our model, i.e. mortality intensities.

As noted earlier in this section, we limit our considerations to one gender and one population only. Actual transactions were based on both genders, but differences in the evolution of male and female death rates are rather small, particularly in view of catastrophic events. This may not be the case for different populations as a possible epidemic or a severe terrorist attack may occur locally. Into a multidimensional version of our model, a complex dependence structure between the catastrophe components can be incorporated. However, for the baseline component, restrictions need to be imposed; for example, independently or fully correlated evolving baseline components will sustain the model's analytical tractability, but empirical investigations are necessary to support such assumptions. Furthermore, the number of parameters will naturally increase.

As mentioned earlier in the text, we primarily focus on the Tartan transaction, which is solely based on US mortality experience. Denoting $\frac{1}{2}\left(\hat{q}_{2005}+\hat{q}_{2004}\right)$ by $i_{0}$, the combined mortality index $i_{t}$ at times $t=2(2007), 3(2008)$ in terms of our model is given by (cp. Equation (2) $)^{30}$

$$
\begin{aligned}
i_{t} & =\frac{1}{2 i_{0}}\left(\hat{q}_{t}+q_{t-1}\right) \\
& =\frac{1}{2 i_{0}} \sum_{x} \omega_{x, m}\left(\left(1-e^{-\int_{0}^{1} \mu(x+s, t-1+s) d s}\right)+\left(1-e^{-\int_{0}^{1} \mu(x+s, t+s) d s}\right)\right) \\
& =\frac{1}{i_{0}}-\sum_{x} \frac{\omega_{x, m}}{2 i_{0}}\left(e^{-\int_{0}^{1} \mu(x+s, t-1+s) d s}+e^{-\int_{0}^{1} \mu(x+s, t+s) d s}\right)
\end{aligned}
$$

In order to determine "the value", i.e. the expected discounted payoff under a certain model parametrization, we need to determine the cash flows of the security. As explained in Section 2.1, the investor is entitled to coupon payments. During the first measurement period, that is the time before the index is calculated for the first time (here $t=2$ ), the coupon payments are not at risk. For the remaining time, interest is only paid on the remaining principal, which is determined according to Equation (3). At maturity, interest for the last period and the remaining principal are disbursed. Table 7 shows the resulting cash flows for a nominal of 1 and spread $s$ within the Tartan transaction. To simplify notation, annual instead of quarterly coupon payments are assumed; $a$ and $d$ denote the attachment and detachment point, respectively. Thus, it is sufficient to determine the (joint) distributions of $i_{2}$ and $i_{3}$ to derive the value as the sum of the expected discounted cash flows.

While there is an approximative method to solve the valuation problem semianalytically, the derivation is computationally involved as it requires numerical methods for inverting multidimensional Fourier/Laplace transforms and the numerical computation of multidimensional integrals (see the Appendix for details). But aside from the computational difficulties, we are faced with a practical constraint: There are only very few market prices available. Naturally, we need at least as many quotes as there are parameters to be calibrated. In particular, for the

\footnotetext{
${ }^{30}$ Note that in comparison to Table 1 , the weights $\omega_{x, m}$ need to be adjusted as we consider only male and single age rather than age group weightings.
} 


\begin{tabular}{|l|l|}
\hline \hline & Cash Flows \\
\hline$t=0$ & -1 \\
$t=1$ & $(\mathrm{LIBOR}+s) 1$ \\
$t=2$ & $(\mathrm{LIBOR}+s) 1$ \\
$t=3$ & $(\mathrm{LIBOR}+s)\left(1-\min \left\{1, \max \left\{\frac{i_{2}-a}{d-a}, 0\right\}\right\}\right)$ \\
& $+\left(1-\min \left\{1, \max \left\{\frac{i_{2}-a}{d-a}, \frac{i_{3}-a}{d-a}, 0\right\}\right\}\right)$ \\
\hline \hline
\end{tabular}

Table 7: Cash flows for the Tartan bonds

Tartan deal there is only a single price available as there is only one (unwrapped) tranche which enables us to only calibrate one parameter implicitly. While we may additionally consider prices of other transactions, as for example the OSIRIS or the Vita III deals, within these securities, the combined mortality index is subject to several different populations (e.g. 60\% France for OSIRIS), and assuming the same evolution for the underlying mortality would be rather harsh without empirical investigations.

Therefore, we limit our considerations to the single Tartan tranche and rely on Monte Carlo simulations for both implicitly calibrating the parameter(s) as well as analyzing and pricing the contracts by simulating the index. This is computationally procurable for only one free parameter and has the additional advantage that we can carry out the computation "exactly" rather than using an approximation. However, when there are more prices available, Monte Carlo simulations may not present a feasible choice for calibrating several parameters at a time and the approximative method from the Appendix may be a valuable alternative.

In the calibration procedure, we are left with the choice of which parameters to fix and which parameter to keep variable since only one free parameter may be included. We choose the expected jump size: On the one hand, the baseline component, by definition, does not reflect the attitude towards catastrophic events, which are most important for the transactions, and is, therefore, set to the conservative parametrization P1 (see Table 4). On the other hand, as we want to interpret a jump of the catastrophe component solely as a catastrophic event, relatively high values for the jump intensity $\lambda$ or very low values for $\kappa$ are not preferable. Hence, we fix $\lambda$ and $\kappa$ to the parameter values 0.074 and 4.6052 which were estimated in Subsection 4.1. Similarly as for pricing insurance contracts, we further need to fix a parametrization for the initial mortality intensity $e^{b x+c}$. We proceed analogously to the last subsection, i.e we derive $\mu\left(x_{0}, 0\right)$ based on the given 2003 population mortality data and the projection method from the American Academy of Actuaries (2002). Now, given the spread level of 300bps for the Class B notes of the Tartan deal, we choose $\zeta$ such that we fit the price - we obtain an expected jump size of $\frac{1}{\zeta}=0.0075726$.

Based on the different parameter choices that we derived in this section, we are now able to price and analyze the CATM bonds from different perspectives.

\section{Results}

For rating agencies and traditional ABS investors, the Probability of Default (PD), that is the probability that the investors' principal will be reduced due to the occurrence of a catastrophic event, as well as the Expected Loss (EL), i.e. the expected percentage of the principal loss, are important comparative statistics. Furthermore, the corresponding spread level leading to an "actuarially fair" contract in the sense that the sum of the expected discounted cash flows equals zero may be of interest. 


\begin{tabular}{|c|c|c|c|c|c|c|}
\hline Scenario & Cl. B & ranche (1 & $10 \%-115 \%)$ & Cl. A : & ranche ( & $5 \%-120 \%)$ \\
\hline & $\mathrm{PD}(\%)$ & $\mathrm{EL}(\%)$ & Spread (bps) & $\mathrm{PD}(\%)$ & EL $(\%)$ & Spread (bps) \\
\hline P1 & 2.4856 & 1.5675 & 61.80 & 0.9162 & 0.5789 & 22.65 \\
\hline P2 & 2.0954 & 1.3223 & 52.53 & 0.7710 & 0.4856 & 19.20 \\
\hline P3 & 1.8896 & 1.1908 & 47.70 & 0.6844 & 0.4346 & 17.29 \\
\hline
\end{tabular}

Table 8: Influence of the Baseline Component

In Table 8, the loss profiles as well as the respective spread levels are displayed for the two tranches within the Tartan deal and the three different historical parametrizations for the baseline component ${ }^{31} \mathrm{P} 1, \mathrm{P} 2$, and $\mathrm{P} 3$.

The results clearly reflect the increased exposure to catastrophic mortality risk of the lower Class B notes in comparison to the more senior Class A notes: All three risk measures are reduced by more than $63 \%$. This does not seem surprising considering the fact that the lower tranche will be completely exhausted if the higher tranche is triggered. Moreover, all three quantities are relatively high for the more conservative parametrization $\mathrm{P} 1$, where only very mild future mortality improvements are assumed. While this general trend also does not seem peculiar, it occurs that the influence of the baseline component is quite pronounced: From parametrization $\mathrm{P} 1$ to $\mathrm{P} 2$, the expected loss is reduced by almost $16 \%$, and from $\mathrm{P} 2$ to $\mathrm{P} 3$ the reduction is still about $10 \%$. This reveals that despite the rather short maturity of three years, the baseline component considerably affects the loss profile. This does not mean that the bond may be triggered by an adverse evolution of the baseline component. In fact, when neglecting the catastrophe component, the default probability is at an almost negligible level for all three parametrizations of the baseline component - but differences in mortality improvements due to the parametrization of the baseline component affect the probability that the tranche is triggered given a "jump" occurred. In Figure 7, the discretized distribution of the index at time $t=3, i_{3}$, is plotted for parametrizations $\mathrm{P} 1$ and $\mathrm{P} 3$. We find that the right tail is only slowly declining in comparison to the left tail. This "skewness" is due to the influence of the catastrophe component, i.e. the influence of the (positive) jumps of the mortality intensity. When comparing the distributions of the index implied by the two baseline parametrizations, it appears that the shape is very similar but that more pronounced mortality improvements lead to a left shift. This means that for higher mortality improvements, catastrophic occurrences may be leveled to the point where they do not lead to a trigger event.

However, the catastrophe component is the more important risk driver for CATM securitizations. In particular, the uncertainties regarding the corresponding parameters have a significant effect: The loss profile of the bonds is very sensitive to changes in all three parameters affecting the catastrophe component. ${ }^{32}$ As indicated in Section 4.1, there are particular problems when trying to find an adequate parametrization for the expected jump size $\frac{1}{\zeta}$ since different observation periods or different demographic structures may yield considerably different out-

\footnotetext{
${ }^{31}$ While discussing the influence of the baseline component, we always assume the following parametrization for the catastrophe component: $\kappa=4.6052, \lambda=0.074, \zeta=357.31$ (cp. Table 5).

${ }^{32}$ See Figures 10, 11, and 12 in the Appendix for sensitivities of the expected tranche loss to changes in $\lambda, \kappa$, and $\frac{1}{\zeta}$, respectively. The basic parametrization of the catastrophe component, which this sensitivity analysis is based on, is again $\kappa=4.6052, \lambda=0.074$, and $\zeta=357.31$ (cp. Table 5).
} 


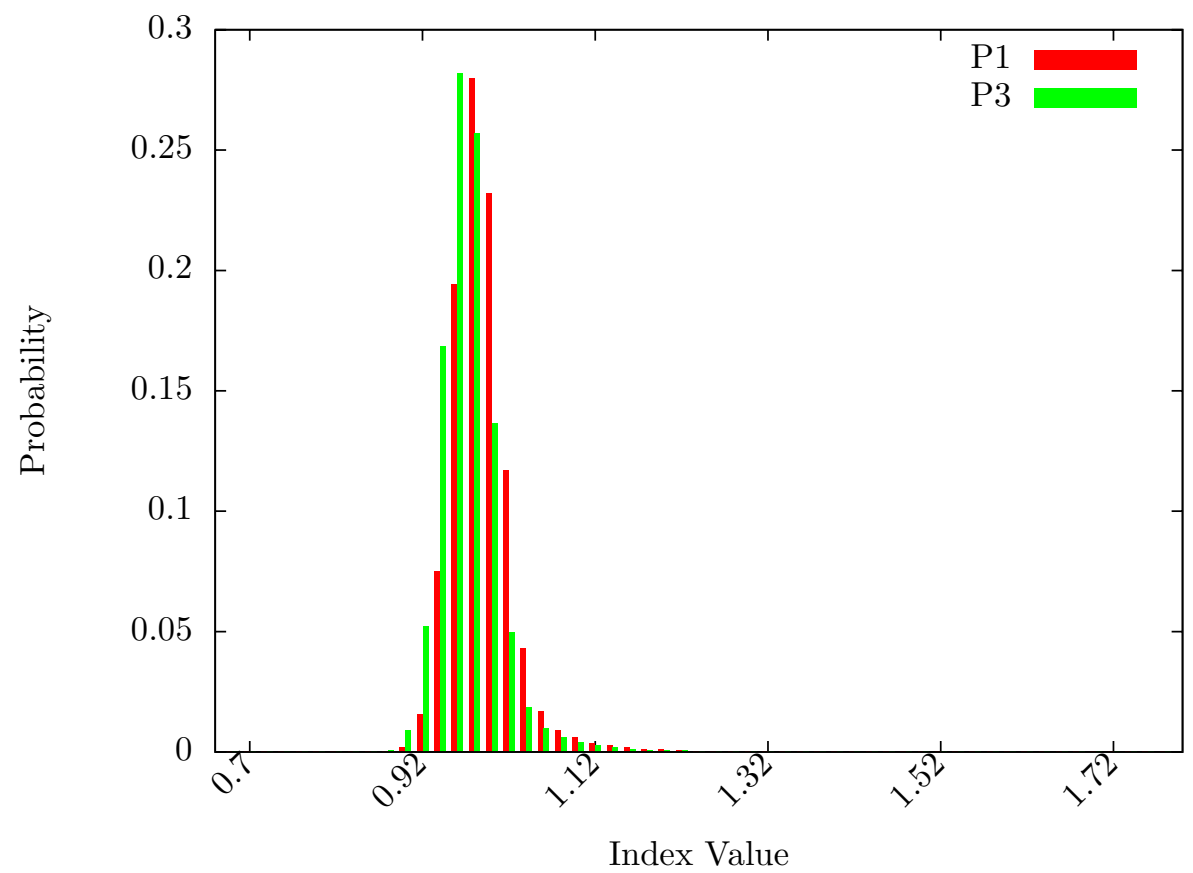

Figure 7: Distribution of the index value

comes of the calibration procedure. In Table 9, comparative statistics for different parametrizations are presented. For the middle choice of the baseline component (P2), aside from risk measures resulting from parameters corresponding to the 2003 demographic structure (P2 and P2'), the results implied by the 1959 demographic structure (P21 and P21') for exponentially as well as Gamma distributed jumps, respectively, are provided. Moreover, the loss profile for the Tartan bonds as quoted from Linfoot (2007) is shown.

We find that there are distinct differences between the two contemplated jump distributions, which emanate from a higher variance of the Gamma distributed jumps. For the utilized data consisting of only five model points, it is arguable whether the additional degree of freedom within the Gamma distribution is essential or even appropriate, but for larger data-sets it may prove necessary in order to obtain a significantly better fit to the empirical distribution. Moreover, in comparison to

\begin{tabular}{|l|cccccc|}
\hline \hline \multirow{2}{*}{ Scenario } & $C l$. B Tranche $(110 \%-115 \%)$ & Cl. A & Tranche $(115 \%$ - 120 \%) \\
\hline & & & & & \\
P21 & 2.6464 & 1.7507 & 70.06 & 1.0854 & 0.7181 & 28.55 \\
P21' & 2.9376 & 2.1615 & 88.16 & 1.5460 & 1.1594 & 47.27 \\
P2 & 2.0954 & 1.3223 & 52.53 & 0.7710 & 0.4856 & 19.20 \\
P2' & 2.4742 & 1.7639 & 71.74 & 1.2208 & 0.8841 & 35.87 \\
Quoted & $\mathbf{0 . 8 8 0 0}$ & $\mathbf{0 . 5 4 0 0}$ & - & $\mathbf{0 . 2 9 0 0}$ & $\mathbf{0 . 1 6 0 0}$ & - \\
& & & & & & \\
\hline \hline
\end{tabular}

Table 9: Influence of the Catastrophe Component 


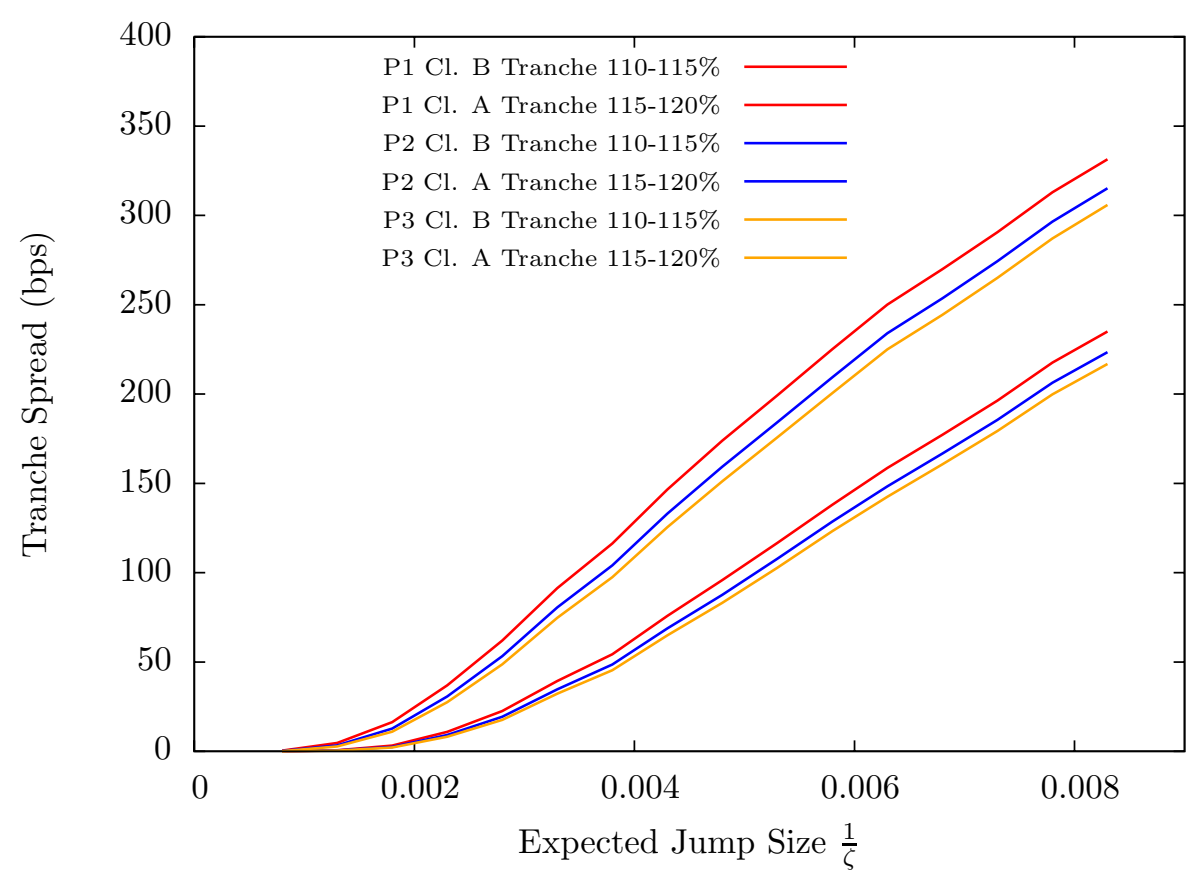

Figure 8: Influence of the expected jump size on the tranche spreads

the jump size distribution, the influence of the population structure appears to be similarly pronounced. The expected loss is reduced by approximately $20 \%$ to $30 \%$ for the different tranches and jump distributions when considering the 2003 opposed to the 1959 demographic structure.

The risk measures from Linfoot (2007) are considerably lower than our results for the 2003 population, but when comparing the results for Class A and B notes as well as the ratios of default probabilities and expected losses within one set of results to our outcomes, the implicit structure is quite similar. This observation indicates that the deviations of our findings in comparison to the "official" quotes from Linfoot (2007) do not result from a distinctive structural difference in the model specification but rather from differences in the considered parametrizations, in particular for the expected jump size $\frac{1}{\zeta}$ and the mean reversion parameter $\kappa$. Regarding the difficulties which come along with the calibration procedure, these deviations indicate that the estimates provided by risk modeling firms should be interpreted carefully by investors and, especially, rating agencies.

Even though the resulting spreads for our parametrizations are presumably higher than the spread levels corresponding to the loss profiles from Linfoot (2007), they are clearly still well below the market level of $300 \mathrm{bps}$. Figure 8 shows the sensitivity of the spread level to changes in the expected jump size $\frac{1}{\zeta}$. For relatively low values, the tranche spreads increase exponentially in the expected jump size. ${ }^{33}$ However, as $\zeta$ decreases, the sensitivity lessens and the curve becomes, ceteris paribus, concave. This peculiarity is due to the structure of the deal: If a jump is large enough such that the complete principal is exhausted, it is not relevant by how much the jump exceeds this critical value. In particular, this means that the differences between the spread levels for the two different tranches decrease with an increasing expected

\footnotetext{
${ }^{33}$ See also Figure 12 in the Appendix for the same observation of the sensitivity of the expected tranche loss.
} 


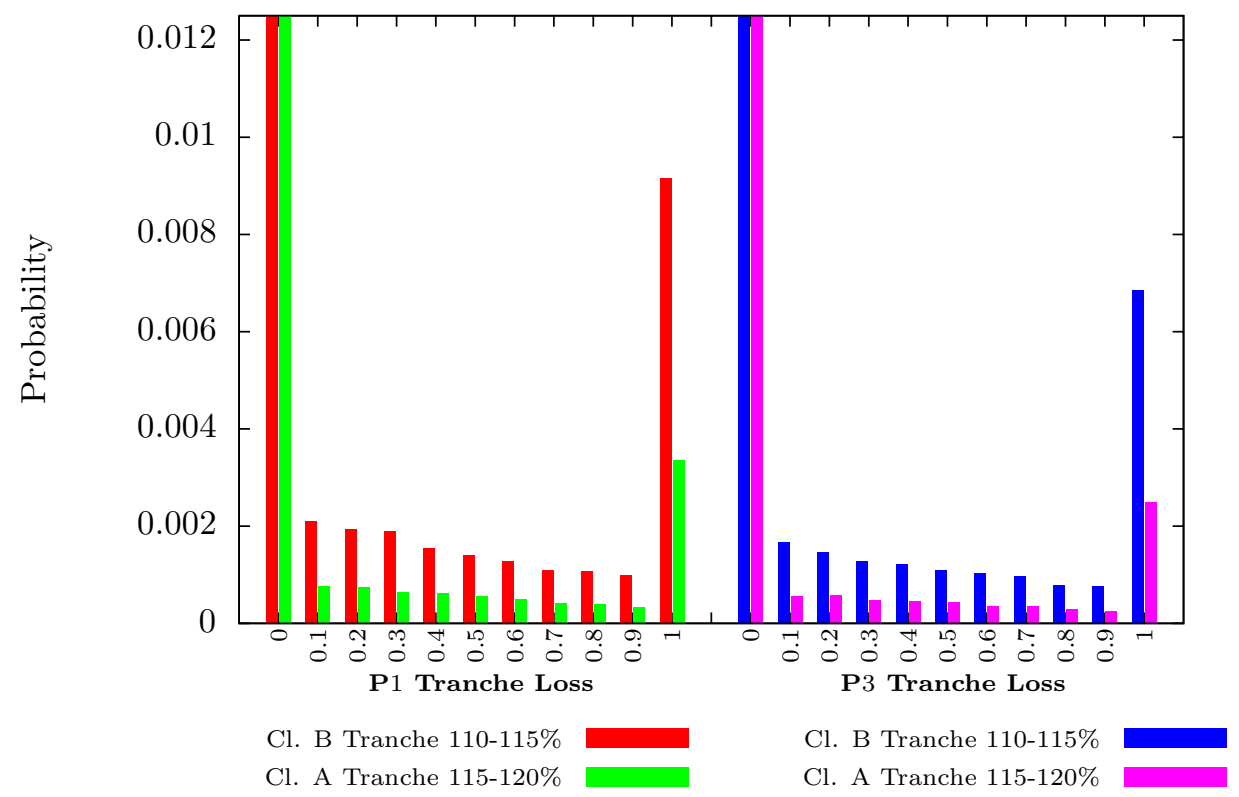

Figure 9: Discretized tranche loss distributions

jump size, as - beyond some critical point - most jumps will fully exhaust both tranches. Thus, we obtain an upper bound of approximately $1200 \mathrm{bps}$ for the spread level since for "infinitely" large jumps, the only question in view is whether a jump occurs or not which is controlled by the jump intensity $\lambda=0.074$.

This structure is similar to the one of CDO tranches. In fact, the tranche loss distributions displayed in Figure 9 have exactly the same shape as for CDOs. ${ }^{34}$ The left tail of the index distribution is attributed to the $0 \%$ loss, whereas the outer right tail is attributed to a full loss. In particular, it is worth noting that the cumulated loss probabilities for the Class A tranche exceeding the $0 \%$ level add up to the full loss probability of the Class B tranche. Moreover, we can again observe the "shift" of the distributions when comparing the histograms for parametrization P1 and P3.

The relatively young history of the CATM market and the unfamiliarity of ABS investors with mortality contingent securities suggest that the spread levels investors can earn within CATM transactions are likely to be above the levels for CDOs with a similar PD and EL. This idea is backed by a comparison of the different CATM deals so far. In Table 10, risk measures and spread levels as implied by the Tartan price (cp. Section 4.3) as well as the quoted market spreads for tranches from the Vita I and the Vita III transactions are displayed. ${ }^{35}$ It appears that the quoted spread level within the Vita I deal was considerably higher than the spread level implied by the Tartan price. This does not seem surprising as Vita I was the first CATM transaction, and consequently the spreads included a considerable novelty premium. Conversely, the actual spread level for the B-II notes of the Vita III transaction is considerably lower than the respective spread implied by Tartan.

\footnotetext{
${ }^{34}$ Note that the probability of a $0 \%$ tranche loss is capped at 0.0125 in Figure 9.

${ }^{35}$ Note that using the same parametrization would suggest that the underlying population is the same for all transactions, which is not the case. Thus, our findings have to be considered with care as e.g. diversification effects are not included.
} 


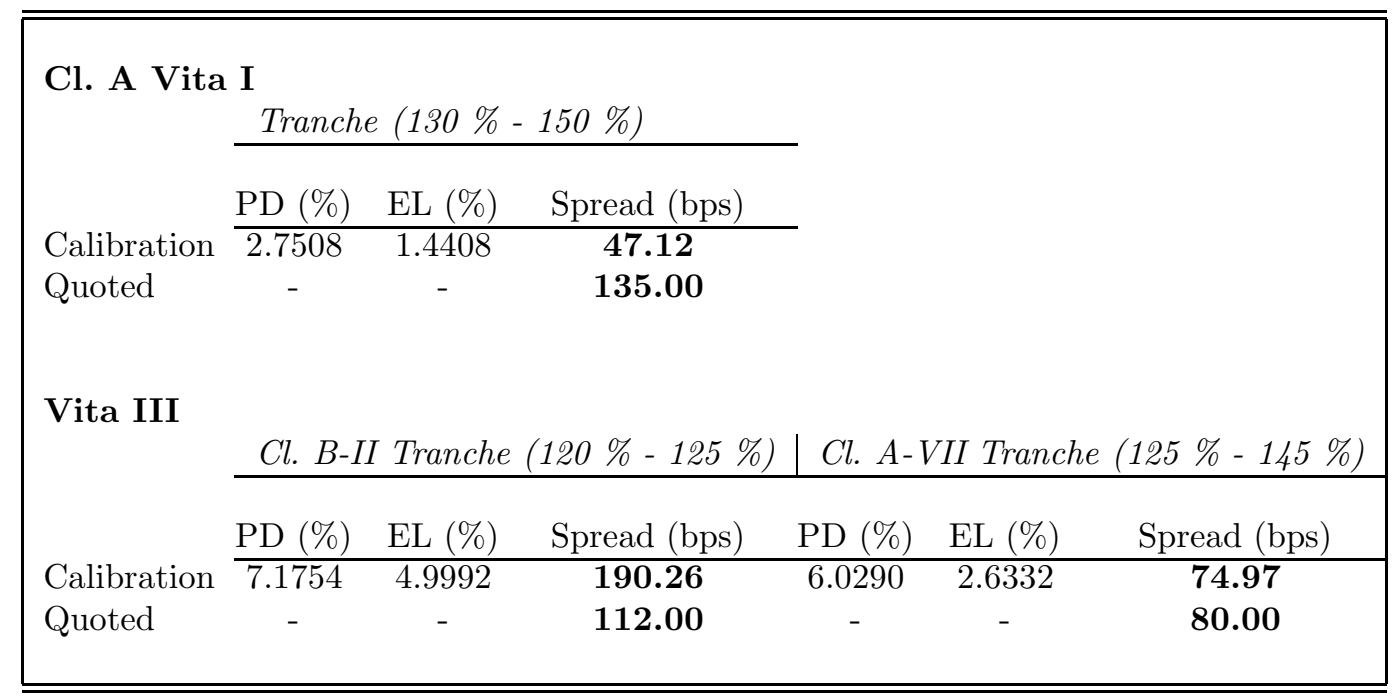

Table 10: Summary of results for risk-adjusted parametrization based on the Tartan tranche

This relationship cannot be observed for the A-VII tranche: Here, the market spread slightly exceeds the Tartan-implied spread. However, the difference of the market spreads between the two Vita tranches seems rather small considering the significantly higher exposure of the Class B-II note to catastrophic occurrences. In particular, if we adjust the expected jump size to match one of the two prices, the other model-endogenous spread will be far from the observed one. However, we only adjust one parameter, namely the expected jump size; as depicted earlier in this section, the sensitivity of the spread level to the expected jump size fades for higher values of $\frac{1}{\zeta}$ (see Figure 8), whereas the spread level decreases exponentially in $\kappa$ and increases almost linearly in the jump intensity $\lambda .{ }^{36}$ Therefore, using our model, it is possible to mimic price structures as observed within the Vita III transaction by simultaneously adjusting several parameters instead of a single one. However, a calibration via Monte Carlo simulations as was carried out in Section 4.3 will become cumbersome. Thus, when calibrating our model to several tranche prices as e.g. within the Osiris or Vita III transaction, the calibration procedure based on the approximative derivation of the index distribution from the Appendix, which is also adverted in Section 4.3, may be advisable.

Table 11 shows the results for the risk-adjusted parametrization based on insurance prices as was explained in Section 4.2. In comparison to the results from the historic parametrizations, we find that all risk measures increase dramatically. Despite the problems with the calibration due to selection effects and differences in the populations considered as described in the foregoing section, these large deviations indicate that insurance prices include considerable margins for adverse mortality evolutions due to occurrences of catastrophic events. In particular, we find that the resulting spread level of approximately 277bps for the Class B notes is only slightly lower than the market spread of 300bps. Disregarding possible flaws, this means that Scottish Re was able to bin the catastrophic mortality risk from their books for less than 23bps, since the 277bps constitute a lower bound for the spread level implied by insurance prices (cp. 4.2). Furthermore, as detailed in Section 2.2 and indicated

\footnotetext{
${ }^{36}$ See Figures 10 and 11 in the Appendix for the sensitivity of the expected tranche loss to changes in the jump intensity and the speed of mean reversion.
} 


\begin{tabular}{|lcccccc|}
\hline \hline & \multicolumn{1}{c}{ Tranche $(110 \%-115 \%)$} & Tranche $(115 \%$ - 120 \%) \\
\cline { 2 - 7 } & & & & & & \\
Calibration & 7.1272 & EL (\%) & Spread (bps) & PD (\%) & EL (\%) & Spread (bps) \\
Quoted & - & - & $\mathbf{2 7 7 . 2 9}$ & 6.2092 & 5.8092 & 239.36 \\
& & & & - & - & - \\
\hline \hline
\end{tabular}

Table 11: Summary of results for risk-adjusted parametrization based on insurance prices

in Table 10, the Tartan deal was priced wider than many other deals, meaning that this difference may have even been smaller for other transactions. Hence, it is even conceivable that within some tighter priced notes, such as the Vita III Class B-II notes, (re)insurers were able to lay off their catastrophic mortality risk by earning rather than paying a premium.

These observations provide us with a possible answer to the question of why the CATM market has grown so quickly over the last years: For ABS investors, CATM transactions provide investment opportunities with a familiar payoff structure, but the spreads one can earn seem to exceed the ones within the credit market, possibly due to considerable novelty premiums. However, the margins for catastrophic mortality risk within insurance prices appear to be, if at all, only slightly lower than the margins within the CATM bonds. Thus, for (re)insurers, CATM transactions seem to provide relatively cheap - or even profitable - means to remove catastrophic mortality risks from their liability side.

In this regard, the Theory of General Economic Equilibrium suggests that it is even possible that a growing CATM market with decreasing spread margins may eventually yield decreasing life insurance premiums, which in turn would induce welfare gains.

\section{Conclusion}

Catastrophe Mortality Bonds are a recent capital market innovation providing insurers and reinsurers with the possibility to transfer catastrophe mortality risk off their balance sheets to capital markets. While the various transactions differ in the composition of the underlying reference population, the basic structure is the same: Based on mortality data as reported by official entities, a combined mortality index is calculated. If this index exceeds a certain level, the bonds will be triggered and the investors' principal will be reduced. In return, investors receive coupon payments on their principal including spread margins for the adopted risk.

So far, there have been five public deals in the market, four of which Swiss Re has been involved in as protection buyer and/or arranger. Since the first deal in late 2003, in which only one tranche with a relatively low risk exposure was issued, the market has developed considerably - more recent deals include several tranches with different seniorities. Moreover, the spectrum of investors has widened substantially: While the first bond was mainly sold within the insurance world or to specialized CAT bond investors, now several tranches are wrapped by monoline insurers, and traditional ABS investors as well as hedge funds have found interest in these securities.

An important role in the arrangement and the execution of these transactions is played by so-called risk modeling firms, who are in charge of the calculation of the 
combined mortality index and the provision of comparative statistics such as default probabilities or expected losses of the securities for investors and rating agencies. Despite the growing market and the increasing bandwidth of investors, so far only one company, the actuarial consultant Milliman, was appointed as the calculation agent for all deals. However, within the last transaction (Vita III), the consulting firm Risk Management Solutions was hired as an adviser by one of the involved monoline insurers. Their modeling approaches differ considerably: While Milliman uses statistical forecasts based on an actuarial model, RMS relies on their expertise regarding pandemic occurrences in a causal modeling approach.

In this article, a time-continuous actuarial model for analyzing and pricing mortality contingent securities is introduced. The model consists of two additive parts: A baseline component, which models the "regular" random fluctuations of mortality over time and is driven by a diffusion, and a catastrophe component governed by a jump process. Due to its affine structure, survival probabilities can be determined analytically up to the solution of ordinary differential equations, and - on this basis - the "classical actuarial toolbox" can be used to determine insurance premiums, for example.

In order to apply this model for analyzing mortality contingent securities, it naturally needs to be calibrated. We provide a detailed discussion of different calibration procedures and resulting parametrizations. In addition to a calibration based on historical data, we derive risk-adjusted parametrizations based on insurance quotes and market prices of catastrophe mortality bonds, respectively.

Our discussion shows that finding adequate parameters based on the data used in practice is very difficult, particularly for the catastrophe component. Therefore, we do not consider a single set of parameters but several parametrizations and conduct detailed sensitivity analyses. We find that the outcomes regarding expected losses and default probabilities of the considered securities differ significantly among the different sets of parameters, which leads to the conclusion that loss profiles as provided by the risk modeling firms have to be considered with care. In particular, the provided risk measures are substantially lower than our results for all considered parametrizations although there are no structural differences in the outcomes, which indicates that the parametrizations used by the calculation agents are rather "optimistic". A collaboration of actuaries and experts in epidemiological research may potentially lead to more reliable results.

Analyzing the loss distribution of the notes, one detects that they look very similar to loss distributions of CDO tranches, suggesting that ABS investors may feel quite comfortable with these securities as they are used to their structure. Moreover, when comparing loss probabilities and expected losses, the risk-return profile of CATM bonds seems to be very attractive. However, one has to keep in mind that the structure of the underlying risk is not alike meaning that comparisons based on low order moments or partial moments may be misleading. Nevertheless, a comparison of the pricing of the different transactions suggests that there is a substantial novelty premium included in the spread margins, which explains the investors' interest in the notes.

Comparing the spread margins to notional margins in term life insurance prices, the differences seem to be rather small. This indicates that insurers and reinsurers can take advantage of the risk transfer at a relatively low cost or even by earning a premium, which may explain the quick growth of the market from the insurer's perspective.

For assessing CATM bonds with more than one underlying population, as a next step, the model can be extended to multiple dimensions. As depicted in Section 4.3, the model structure and, in particular, the analytic properties will remain the same under certain assumptions on the dependence of the respective baseline components. For the securities considered, the introduced model performs very well for ana- 
lyzing and pricing, and this article provides valuable insights into the market for catastrophe mortality bonds.

\section{References}

American Academy of Actuaries, 2002. Final Report of the American Academy of Actuaries Commissioners Standard Ordinary Task Force. Presented in Draft to the National Association of Insurance Commissioners Life and Health Actuarial Task Force, Philadelphia, PA .

Bantwal, V.J., Kunreuther, H.C., 1999. A Cat Bond Premium Puzzle?. The Journal of Psychology and Financial Markets, 1: 76-91.

Bauer, D., Russ, J., 2006. Pricing Longevity Bonds using Implied Survival Probabilities. Working Paper, Ulm University and Institut für Finanz- und Aktuarwissenschaften. Available at: www.mortalityrisk.org.

Biffis, E., 2005. Affine processes for dynamic mortality and actuarial valuation. Insurance: Mathematics and Economics, 37:443-468.

Biffis, E., Denuit, M., Devolder, P., 2005. Stochastic Mortality under Measure Change. Working Paper, CASS Business School and Université Catholique de Louvain.

Bingham, N., Kiesel, R., 2003. Risk-Neutral Valuation. Springer Finance. Springer, London.

Björk, T., 1999. Arbitrage Theory in Continuous Time. Oxford University Press, Oxford, UK.

Bowers, N.L., Gerber, H.U., Hickman, J.C., Jones, D.A., Nesbitt, C.J., 1997. Actuarial Mathematics. The Society of Actuaries, Schaumburg, Il, USA.

Cairns, A.J., Blake, D., Dowd, K., 2006. Pricing Death: Frameworks for the Valuation and Securitization of Mortality Risk. ASTIN Bulletin, 36:79-120.

Cont, R., Tankov, P., 2004. Financial Modelling With Jump Processes. Chapman \& Hall/CRC, Boca Raton, Fl.

Cowley, A., Cummins, J.D., 2005. Securitization of Life Insurance Assets and Liabilities. The Journal of Risk and Insurance, 72: 193-226.

Cox, J.C., Ingersoll, J.E., Ross, S.A.., 1985. A Theory of the Term Structure of Interest Rates. Econometrica, 53: 385-407.

Cox, S.H., Lin, Y., Wang, S., 2006. Multivariate Exponential Tilting and Pricing Implications for Mortality Securitization. The Journal of Risk and Insurance, 73: 719-736.

Cox, S.H., Pedersen, H.W., Fairchild, J.R., 2000. Economic aspects of securitization of risk. ASTIN Bulletin, 30: 157-193.

Cummins, J.D., 2006. Should the Government Provide Insurance for Catastrophes?. Federal Reserve Bank of St. Louis Review, 88: 337-379.

Culp, C.L., 2006. Structured Finance and Insurance. Wiley, Hoboken, NJ. 
Dahl, M., 2004. Stochastic mortality in life insurance: market reserves and mortality-linked insurance contracts. Insurance: Mathematics and Economics, 35: $113-136$.

Dahl, M., Melchior, M., Møller, T., 2006. On systematic mortality risk and riskminimization with survivor swaps. Working Paper, Nordea Markets and PFA Pension, Copenhagen. Available at: www.mortalityrisk.org.

Deacon, J., 2004. Global securitisation and CDOs. Wiley, Hoboken, N.J..

Doherty, N., 1997. Financial Innovations in the Management of Catastrophic Risk. Journal of Applied Corporate Finance, 10: 84-95.

Duffie, D., Kan, R., 1996. A yield-factor model of interest rates. Mathematical Finance, 6: 379-406

Duffie, D., Pan, J., Singleton, K., 2000. Transform analysis and asset pricing for affine jump-diffusions. Econometrica, 68: 1343-1376.

Equitable, 2004. Equitable Accumulator Product Prospectus. The Equitable Life Assurance Society of the United States, New York.

Euroweek, 2006. Capital markets shield AXA from extreme mortality risk. Euroweek, 978(11/2006): 62 .

Froot, K.A., 2001. The market for catastrophic risk: a clinical examination. Journal of Financial Economics, 60.

Gompertz, B., 1825. On the Nature of the Function Expressive of the Law of Human Mortality, and on a New Mode of Determining the Value of Life Contingencies. Philosophical Transactions of the Royal Society of London, 115: 513-585.

Group of Thirty, 2006. Reinsurance and International Financial Markets. Group of Thirty, Washington, DC.

International Financing Review, 2006. Mortality Bond issue upsized. International Financing Review, 1656(11/04/2006): 40.

Jeffrey, P., 2006. A Practitioner's Guide to Securitisation. City \& Financial Publishing, Surrey.

Keyfitz, N., 1985. Applied Mathematical Demography. 2nd Ed., Springer, New York.

Kristen, R., 2007. Design und Bewertung von Sterblichkeitsderivaten. Diploma Thesis, Ulm University.

Lando, D., 1998. On Cox processes and credit risky securities. Review of Derivatives Research, 2: 99-120.

Lee, R., 2001. Predicting Human Longevity. Science, 292: 1654-1655.

Lee, J.-P., Yu, M.-T., 2002. Pricing Default-Risky CAT bonds with Moral Hazard and Basis Risk. The Journal of Risk and Insurance, 69: 25-44.

Lin, Y., Cox, S., 2005. Securitization of Mortality Risks in Life Annuities. The Journal of Risk and Insurance, 72: 227-252.

Lin, Y., Cox, S.H., 2006. Securitization of Catastrophe Mortality Risks. Working Paper, Youngstown State University and Georgia State University. 
Linfoot, A., 2007. Financing Catastrophic Risk: Mortality Bond Case Study. Presentation on behalf of Scottish Re, 02/14/2007.

Logisch, H.H., 2007. A case study about Catastrophe Mortality Securitization. Diploma Thesis, Ulm University and Commerzbank AG.

Luciano, E., Vigna, E., 2005. Non mean reverting affine processes for stochastic mortality. ICER Applied Mathematics Working Paper Series, Working Paper no. $4 / 2005$.

Milevsky, M.A., Promislow, S.D., 2001. Mortality Derivatives and the Option to Annuitize. Insurance: Mathematics and Economics, 29:299 - 318.

Miltersen, K.R., Persson, S.A., 2005. Is mortality dead? Stochastic force of mortality determined by no arbitrage. Working Paper, University of Bergen.

Muermann, A., 2003. Actuarially Consistent Valuation of Catastrophe Derivatives. The Wharton Financial Institution Center Working Paper Series, 03-18. University of Pennsylvania.

Niehaus, G., 2002. The allocation of catastrophe risk. Journal of Banking and Finance, 26: 585-596.

Oeppen, J., Vaupel, J.W., 2002. Broken Limits to Life Expectancy. Science, 269: 1029-1031.

Olivieri, A., 1987. Uncertainty in mortality projections: an actuarial perspective. Insurance: Mathematics and Economics, 29: 231-245.

Olshansky, S.J., Carnes, B.S., Désquelles, A., 2001. Demography: Prospects for Human Longevity. Science, 291: 1491-1492.

Petrella, G., 2004. An extension of the Euler Laplace transform inversion algorithm with applications in option pricing. Operations Research Letters, 32: 380-389.

Pitacco, E. , 2004. Longevity Risk in Living Benefits. Developing an Annuity Market in Europe, Fornero, E. and Luciano, E. Eds. Edward Elgar, Cheltenham.

Schrager, D., 2006. Affine stochastic mortality. Insurance: Mathematics and Economics, 38:81-97.

Standard and Poor's, 2006. Ratings Raised On Vita Capital II's CatastropheIndexed Notes.

Walter, U., 1996. Die Bewertung von Zinsoptionen. Gabler Verlag, Wiesbaden.

Wang, S.S. , 2000. A Class of Distortion Operations for Pricing Financial and Insurance Risks. The Journal of Risk and Insurance, 67: 15-36.

Wang, S.S. , 2002. A Universal Framework for Pricing Financial and Insurance Risks. ASTIN Bulletin, 32: 213-234.

Young, V.R., 2004. Pricing in an Incomplete Market with an Affine Term Structure. Mathematical Finance, 14: 359-381. 


\section{Appendix}

\section{Derivation of Equation (8)}

Equation (8) is a special case of the far more general result from Duffie et al. (2000), Proposition 1. However, for the sake of completeness, we shortly re-derive it for our specification.

Equations (5) and (6) together with the independence of $\Gamma$ and $Y$ yield

$$
\begin{aligned}
{ }_{T-t} p_{x_{0}+t}^{(t)} & =E\left[\exp \left\{-\int_{t}^{T} \mu\left(x_{0}+s, Y_{s}, \Gamma_{s}\right) d s\right\} \mid \mathcal{G}_{t}\right] \\
& =E\left[\exp \left\{-\int_{t}^{T} Y_{s} e^{b\left(x_{0}+s\right)+c}+\Gamma_{s} d s\right\} \mid \mathcal{G}_{t}\right] \\
& =E\left[\exp \left\{-\int_{t}^{T} Y_{s} e^{b\left(x_{0}+s\right)+c} d s\right\} \mid \mathcal{G}_{t}\right] E\left[\exp \left\{-\int_{t}^{T} \Gamma_{s} d s\right\} \mid \mathcal{G}_{t}\right]
\end{aligned}
$$

so we can consider the factors individually.

For the second part, we assume

$$
\begin{aligned}
E\left[\exp \left\{-\int_{0}^{T} \Gamma_{s} d s\right\} \mid \mathcal{G}_{t}\right] & =\exp \left\{-\int_{0}^{t} \Gamma_{s} d s\right\} E\left[\exp \left\{-\int_{t}^{T} \Gamma_{s} d s\right\} \mid \mathcal{G}_{t}\right] \\
& \stackrel{!}{=} \exp \left\{-\int_{0}^{t} \Gamma_{s} d s\right\} \exp \left\{\hat{u}(T-t)+\hat{v}(T-t) \Gamma_{t}\right\} \\
& =: \hat{f}\left(t, \Gamma_{t}\right),
\end{aligned}
$$

where $\hat{u}(0)=\hat{v}(0)=0$. An application of Itô's formula for jump processes (cp. e.g. Cont and Tankov (2004), Proposition 8.13) yields

$d \hat{f}\left(t, \Gamma_{t}\right)=\hat{f}\left(t, \Gamma_{t-}\right)\left(-\Gamma_{t}\left(1+\hat{v}^{\prime}(T-t)+\hat{v}(T-t) \kappa\right)-\hat{u}^{\prime}(T-t)+\lambda\left(\frac{\hat{v}(T-t)}{\zeta-\hat{v}(T-t)}\right)\right) d t+d M_{t}$,

where $\left(M_{t}\right)$ is a martingale. Since $\hat{f}\left(t, \Gamma_{t}\right)$ should be a martingale and by the so-called "affine matching principle" (see Duffie and Kan (1996)), we obtain the following ODEs for $\hat{u}$ and $\hat{v}$ :

$$
\begin{aligned}
& \hat{u}^{\prime}(x)=\frac{\lambda \hat{v}(x)}{\zeta-\hat{v}(x)}, \hat{u}(0)=0 \\
& \hat{v}^{\prime}(x)=-\kappa \hat{v}(x)-1, \hat{v}(0)=0 .
\end{aligned}
$$

As can be easily checked, the unique solution to the latter ODE is

$$
\hat{v}(x)=\frac{-1}{\kappa}\left(1-e^{-\kappa x}\right),
$$

and plugging it into the first ODE and computing the integral leads to

$$
\hat{u}(x)=\frac{-\lambda x}{\zeta \kappa+1}+\frac{-\lambda \zeta}{\zeta \kappa+1} \log \left\{1+\frac{1}{\zeta \kappa}\left(1-e^{-\kappa x}\right)\right\} .
$$

Analogously, for the first part we assume

$$
\begin{aligned}
& E\left[\exp \left\{-\int_{0}^{T} Y_{s} e^{b\left(x_{0}+s\right)+c} d s\right\} \mid \mathcal{G}_{t}\right] \\
= & \exp \left\{-\int_{0}^{t} Y_{s} e^{b\left(x_{0}+s\right)+c} d s\right\} E\left[\exp \left\{-\int_{t}^{T} Y_{s} e^{b\left(x_{0}+s\right)+c} d s\right\} \mid \mathcal{G}_{t}\right] \\
\stackrel{!}{=} & \exp \left\{-\int_{0}^{t} Y_{s} e^{b\left(x_{0}+s\right)+c} d s\right\} \exp \left\{u(T-t)+v(T-t) Y_{t} e^{b\left(x_{0}+t\right)+c}\right\},
\end{aligned}
$$

where $v(0)=u(0)=0$, and again by an application of Itô's Lemma for Itô processes (cp. e.g. Bingham and Kiesel (2003), Theorem 5.6.2) and "matching" the coefficients as above, we obtain the ODEs

$$
\begin{aligned}
& u^{\prime}(x)=\alpha \beta v(x) e^{b\left(x_{0}+T-x\right)+c}, u(0)=0 \\
& v^{\prime}(x)=-1-(\alpha-b) v(x)-\frac{1}{2} \sigma^{2} v^{2}(x) e^{b\left(x_{0}+T-x\right)+c}, v(0)=0 .
\end{aligned}
$$

Plugging $u, v$ and $\hat{u}, \hat{v}$ back into Equations (16) and (15), respectively, and putting the factors back together finally results in Equation (8). 


\section{Derivation for the estimators of $\alpha$ and $\sigma$ in Table 4}

Discretizing the SDE for $Y$ from Equation (7) yields

$$
Y_{t+1}-Y_{t}=\alpha\left(\beta-Y_{t}\right)+\sigma \sqrt{Y_{t}} N_{t},
$$

where $N_{t}, t=0, \ldots, T-1=43$ are independent $N(0,1)$-distributed random variables. Therefore,

$$
\sigma N_{t}=\frac{Y_{t+1}}{\sqrt{Y_{t}}}-\sqrt{Y_{t}}+\alpha \sqrt{Y_{t}}-\frac{\alpha \beta}{\sqrt{Y_{t}}},
$$

and the standard estimators for the mean and the variance (ideally) give

$$
\begin{aligned}
\frac{1}{T} \sum_{t=0}^{T-1} \frac{Y_{t+1}}{\sqrt{Y_{t}}}-\sqrt{Y_{t}}+\alpha \sqrt{Y_{t}}-\frac{\alpha \beta}{\sqrt{Y_{t}}} \stackrel{!}{=} \quad 0 \quad \text { and } \\
\frac{1}{T-1} \sum_{t=0}^{T-1}\left(\frac{Y_{t+1}}{\sqrt{Y_{t}}}-\sqrt{Y_{t}}+\alpha \sqrt{Y_{t}}-\frac{\alpha \beta}{\sqrt{Y_{t}}}\right)^{2} \stackrel{!}{=} \sigma^{2},
\end{aligned}
$$

respectively. As $\beta$ is given, solving the first equation for $\alpha$ yields the estimator

$$
\hat{\alpha}=\frac{\sum_{t=0}^{T-1} \frac{Y_{t+1}}{\sqrt{Y_{t}}}-\sqrt{Y_{t}}}{\sum_{t=0}^{T-1} \frac{\beta}{\sqrt{Y_{t}}}-\sqrt{Y_{t}}},
$$

and plugging $\hat{\alpha}$ back into the second equation gives the estimator for $\sigma$,

$$
\hat{\sigma}=\sqrt{\frac{1}{T-1} \sum_{t=0}^{T-1}\left(\frac{Y_{t+1}}{\sqrt{Y_{t}}}-\sqrt{Y_{t}}+\hat{\alpha} \sqrt{Y_{t}}-\frac{\hat{\alpha} \beta}{\sqrt{Y_{t}}}\right)^{2}} .
$$

\section{Approximative derivation of the joint distribution of $i_{2}$ and $i_{3}$}

By Equation (14), we have

$$
\begin{aligned}
i_{t}= & \frac{1}{i_{0}}-\sum_{\text {all } x} \frac{\omega_{x, m}}{2 i_{0}}\left(e^{-\int_{0}^{1} \mu(x+s, t-1+s) d s}+e^{-\int_{0}^{1} \mu(x+s, t+s) d s}\right) \\
\underset{\text { Taylor }}{\approx} & \frac{1}{i_{0}}-\sum_{\text {all } x} \frac{\omega_{x, m}}{2 i_{0}}\left(\left(1-\int_{0}^{1} \mu(x+s, t-1+s) d s\right)+\left(1-\int_{0}^{1} \mu(x+s, t+s) d s\right)\right) \\
= & \sum_{\text {all } x} \frac{\omega_{x, m}}{2 i_{0}} \int_{0}^{1} \Gamma_{t-1+s} d s+\sum_{\text {all } x} \frac{\omega_{x, m}}{2 i_{0}} \int_{0}^{1} \Gamma_{t+s} d s \\
& +\sum_{\text {all } x} \frac{\omega_{x, m}}{2 i_{0}} \int_{0}^{1} Y_{t-1+s} e^{b(x+s)+c} d s+\sum_{\text {all } x} \frac{\omega_{x, m}}{2 i_{0}} \int_{0}^{1} Y_{t+s} e^{b(x+s)+c} d s \\
=\quad & \frac{1}{2 i_{0}}\left(\int_{t-1}^{t} \Gamma_{s} d s+\int_{t}^{t+1} \Gamma_{s} d s\right)+\int_{t-1}^{t} Y_{s} e^{b s} d s \sum_{\text {all } x} \frac{\omega_{x, m}}{2 i_{0}} e^{b(x-t+1)+c} \\
& +\int_{t}^{t+1} Y_{s} e^{b s} d s \sum_{\text {all } x} \frac{\omega_{x, m}}{2 i_{0}} e^{b(x-t)+c},
\end{aligned}
$$

where we used the simple Taylor expansion $e^{x}=(1+x)$ for $x$ close to one. As the relevant quantities, in particular for the most relevant ages, are very small, the approximation will be close. In order to compute the (joint) distribution of $i_{2}$ and $i_{3}$, we need to compute the joint distributions of the random variables

$$
\begin{aligned}
\text { Continuous Part : } & \Xi_{1}:=\int_{0}^{1} Y_{s} e^{b s} d s, \Xi_{2}:=\int_{1}^{2} Y_{s} e^{b s} d s, \Xi_{3}:=\int_{2}^{3} Y_{s} e^{b s} d s \quad\left(Y_{0}>0\right) ; \\
\text { Jump Part }: & \Lambda_{1}=\int_{0}^{1} \Gamma_{s} d s, \Lambda_{2}=\int_{1}^{2} \Gamma_{s} d s, \Lambda_{3}=\int_{2}^{3} \Gamma_{s} d s \quad\left(\Gamma_{0}=0\right) .
\end{aligned}
$$

As the jump part and the continuous part are independent, it is sufficient to derive the joint densities for $\Xi_{1}, \Xi_{2}$, and $\Xi_{3}$ as well as for $\Lambda_{1}, \Lambda_{2}$, and $\Lambda_{3}$, respectively. The joint density will be given by the product of the two densities. We will focus on the continuous part, but the jump part may be considered analogously. 
It is important to note that $\Xi_{1}, \Xi_{2}$, and $\Xi_{3}$ are independent given $Y_{1}$ and $Y_{2}$. Therefore, for Borel sets $B_{1}, B_{2}, B_{3}, \tilde{B}_{1}, \tilde{B}_{2}$ we have

$$
\begin{aligned}
& P\left(\Xi_{1} \in B_{1}, Y_{1} \in \tilde{B}_{1}, \Xi_{2} \in B_{2}, Y_{2} \in \tilde{B}_{2}, \Xi_{3} \in B_{3}\right) \\
= & P\left(\Xi_{1} \in B_{1}, \Xi_{2} \in B_{2}, \Xi_{3} \in B_{3} \mid Y_{1} \in \tilde{B}_{1}, Y_{2} \in \tilde{B}_{2}\right) P\left(Y_{1} \in \tilde{B}_{1}, Y_{2} \in \tilde{B}_{2}\right) \\
= & P\left(\Xi_{1} \in B_{1} \mid Y_{1} \in \tilde{B}_{1}, Y_{2} \in \tilde{B}_{2}\right) P\left(\Xi_{2} \in B_{2} \mid Y_{1} \in \tilde{B}_{1}, Y_{2} \in \tilde{B}_{2}\right) \\
& P\left(\Xi_{3} \in B_{3} \mid Y_{1} \in \tilde{B}_{1}, Y_{2} \in \tilde{B}_{2}\right) P\left(Y_{1} \in \tilde{B}_{1}, Y_{2} \in \tilde{B}_{2}\right) \\
= & P\left(\Xi_{1} \in B_{1}, Y_{1} \in \tilde{B}_{1}\right) P\left(\Xi_{2} \in B_{2}, Y_{2} \in \tilde{B}_{2} \mid Y_{1} \in \tilde{B}_{1}\right) P\left(\Xi_{3} \in B_{3} \mid Y_{2} \in \tilde{B}_{2}\right) .
\end{aligned}
$$

So, the joint density $f_{\Xi_{1}, \Xi_{2}, \Xi_{3}}(x, y, z)$ can be derived from the (conditional) densities $f_{\Xi_{1}, Y_{1}}(x, y), f_{\Xi_{2}, Y_{2} \mid Y_{1}}(x, y \mid z)$, and $f_{\Xi_{3} \mid Y_{2}}(x \mid y)$ by

$$
f_{\Xi_{1}, \Xi_{2}, \Xi_{3}}(x, y, z)=\int_{\mathbb{R}} \int_{\mathbb{R}} f_{\Xi_{1}, Y_{1}}(x, u) f_{\Xi_{2}, Y_{2} \mid Y_{1}}(y, v \mid u) f_{\Xi_{3} \mid Y_{2}}(z \mid v) d u d v .
$$

Since we are working in an affine framework, we can derive the (joint) Laplace or Fourier transforms for $\Xi_{1}, Y_{1}$, for $\Xi_{2}, Y_{2}$ given $Y_{1}$, and for $\Xi_{3}$ given $Y_{2}$ analytically up to the solution of ODEs similarly to the derivation of Equation 8 above (see Duffie et al. (2000) for details). From these, we can derive the corresponding densities by inverting the respective transform (see e.g. Petrella (2004)), and compute the joint density of $f_{\Xi_{1}, \Xi_{2}, \Xi_{3}}(x, y, z)$ as above; thus, we are given the (joint) distribution of $i_{2}, i_{3}$ as deterministic functions of $\Xi_{1}, \Xi_{2}$, and $\Xi_{3}$.

\section{Additional Figures}

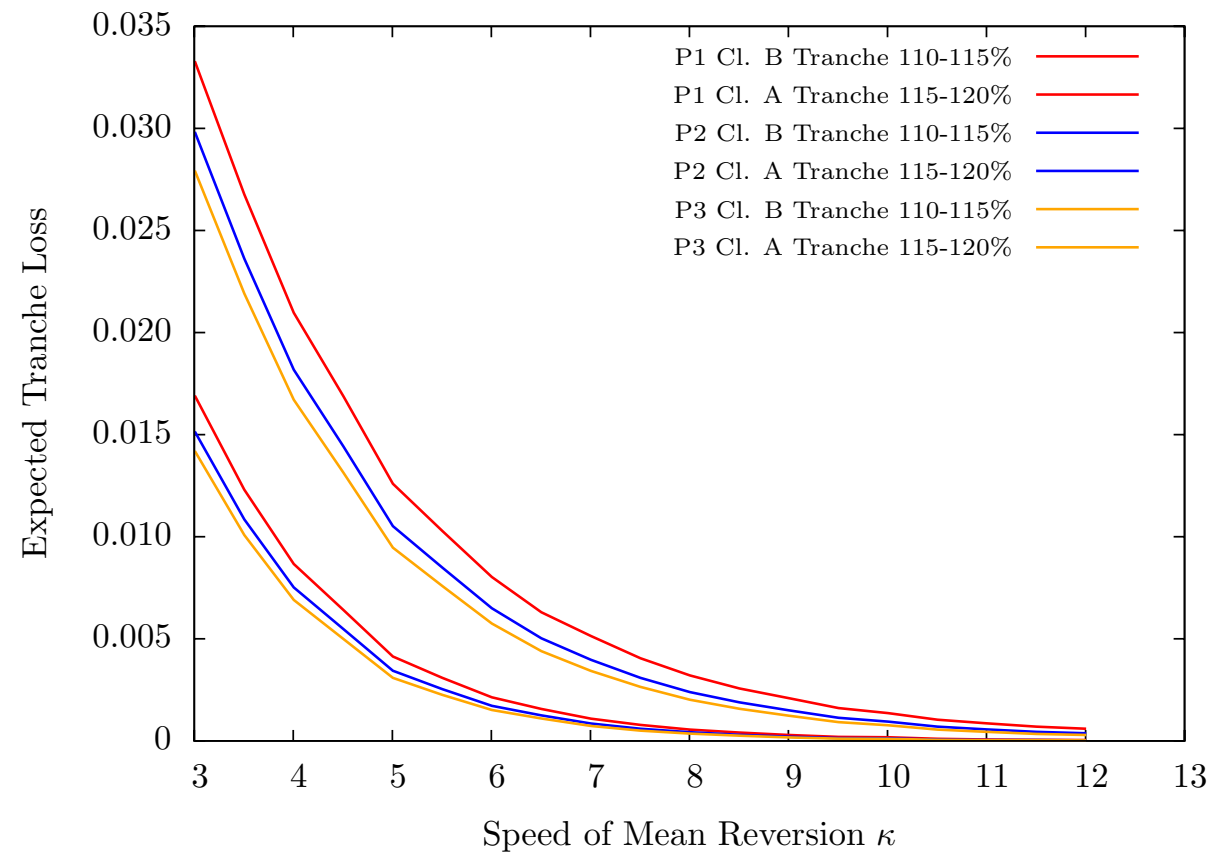

Figure 10: Influence of the speed of mean reversion $\kappa$ on the expected tranche loss 


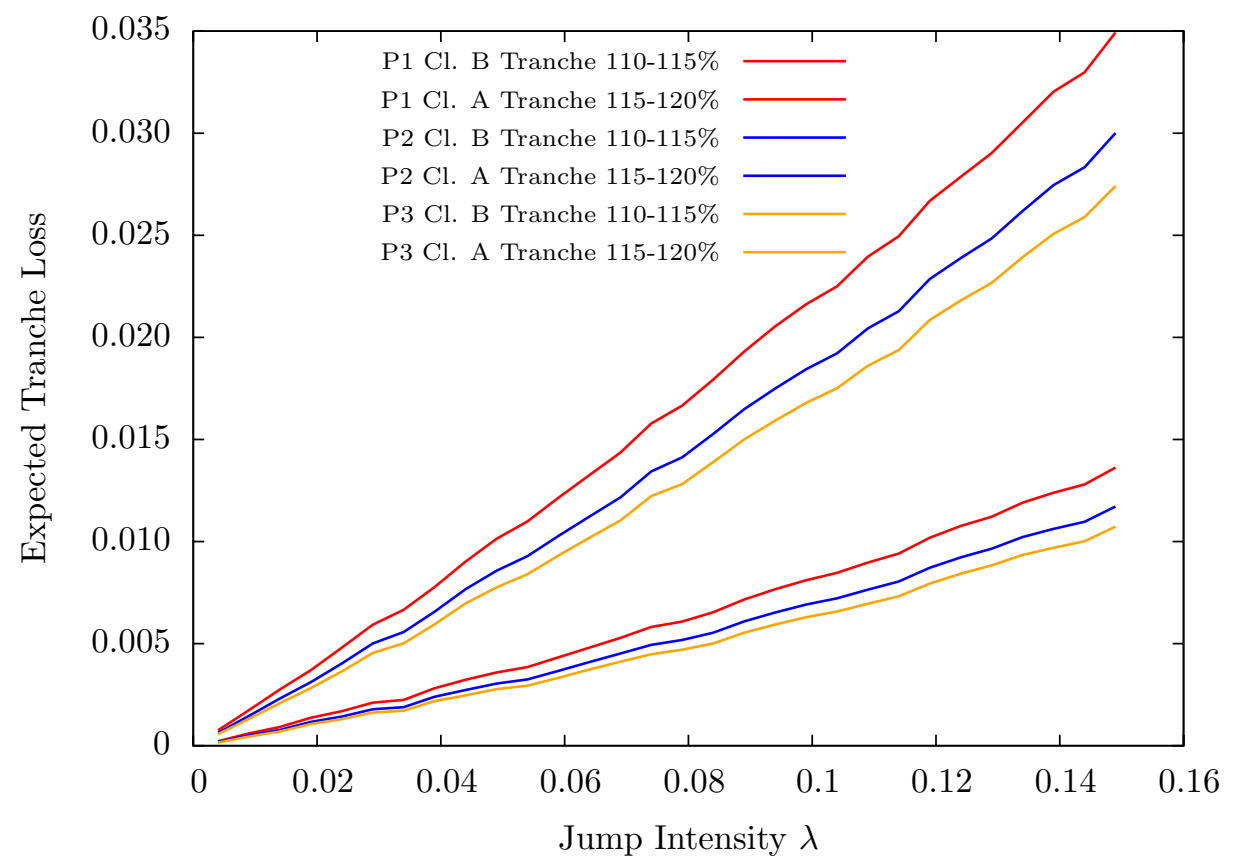

Figure 11: Influence of the jump intensity $\lambda$ on the expected tranche loss

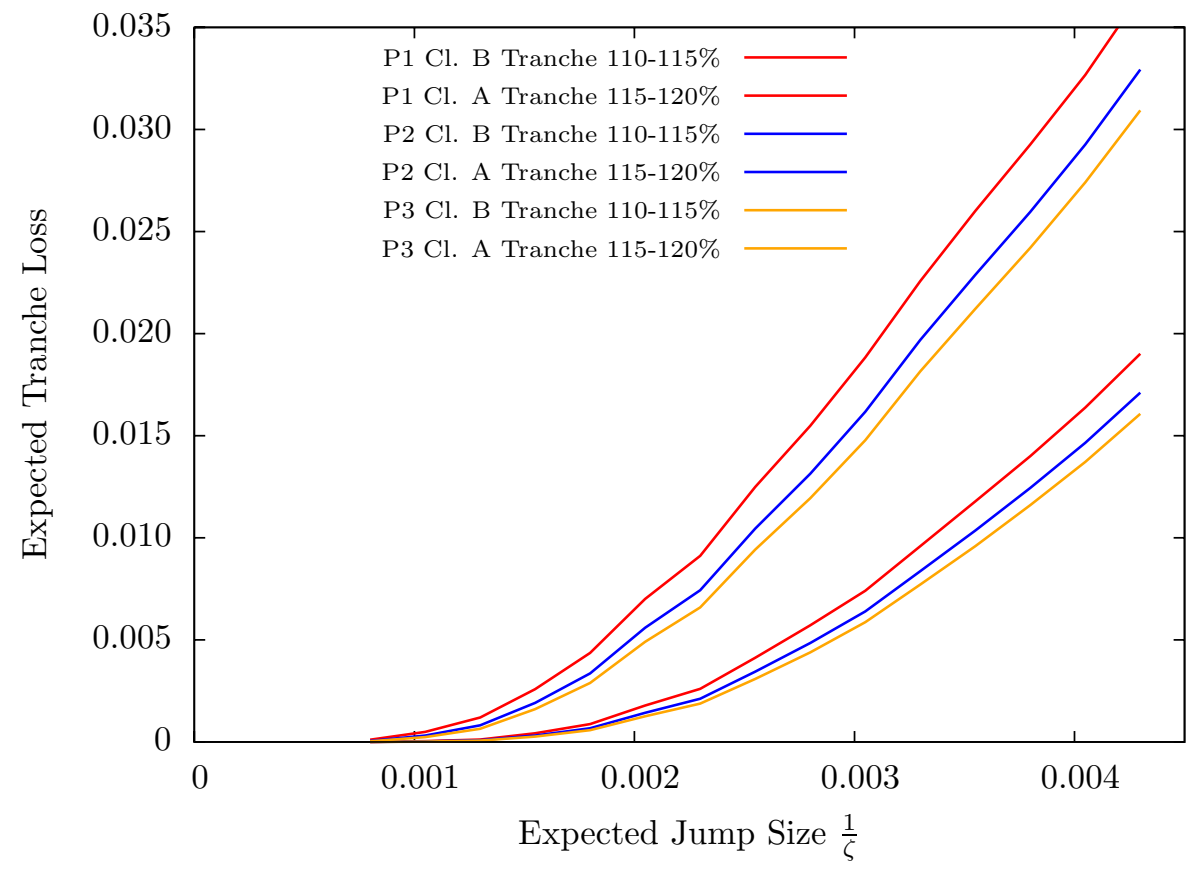

Figure 12: Influence of the expected jump size on the expected tranche loss 